\title{
Mutations decouple proton transfer from phosphate cleavage in the dUTPase catalytic reaction
}

\author{
Anna Lopata ${ }^{\mathrm{a},{ }^{\dagger}}$, Pablo G. Jambrina ${ }^{\mathrm{b}}$, Pankaz K. Sharma ${ }^{\text {c, }}$, Bernard R. Brooks $^{\mathrm{d}}$, Judit Toth ${ }^{\mathrm{a}{ }^{*}}$, Beata G. \\ Vertessy $^{\mathrm{a}, \mathrm{e}^{*}}$ and Edina Rosta ${ }^{\mathrm{b} *}$ \\ ${ }^{a}$ Institute of Enzymology, Research Centre for Natural Sciences, Hungarian Academy of Sciences, \\ Budapest,H1113, Hungary \\ ${ }^{b}$ Department of Chemistry, King's College London, London, SE1 1DB, United Kingdom \\ ${ }^{c}$ College of Pharmacy, Graduate School of Pharmaceutical Sciences, Ewha Womans University, Seoul \\ 120-750 Korea \\ ${ }^{d}$ Laboratory of Computational Biology, National Heart, Lung, and Blood Institute, National Institutes \\ of Health, Rockville, Maryland 20892-9314, United States \\ ${ }^{e}$ Department of Applied Biotechnology and Food Science, Budapest University of Technology and \\ Economics, H1111 Hungary \\ ${ }^{\dagger}$ Present Address: Anna Lopata, Laboratory of Molecular Physiology, National Heart, Lung, and \\ Blood Institute, National Institutes of Health, Bethesda, MD, 20892, USA and Astbury Centre for \\ Structural Molecular Biology, Faculty of Biological Sciences, University of Leeds, Leeds, LS2 9JT, \\ UK \\ ${ }^{t}$ Present Address: Pankaz K. Sharma, Department of Chemistry, Cotton College State University, \\ Guwahati 781001, India \\ "tothj@enzim.hu,Vertessy.Beata@ttk.mta.hu,Edina.Rosta@kcl.ac.uk
}

\begin{abstract}
Most enzymes present a catalytic mechanism where one or more proton transfer events occur coupled tightly together with the enzymatic chemical reaction. We show here that inactivating mutations decouple this proton transfer step from the phosphate cleavage reaction in dUTPase. Homotrimeric dUTPase enzymes catalyze the hydrolysis of dUTP to dUMP and pyrophosphate, using largely similar structural and functional groups as most AAA+ enzymes. dUTPases typically use a single $\mathrm{Mg}^{2+}$ ion as a cofactor in the active site that is formed by direct protein-protein contacts including all three protomers. Here we focus on the C-terminal arm structural motif, which has sequence and functional similarities to P-loop motifs, and is required for catalysis. In this work, we have studied the functional roles of the C-terminal arm in ligand binding and catalysis by using QM/MM (quantum mechanics/molecular mechanics) calculations in conjunction with site-directed mutagenesis experiments. We also present a new method to assess the metal ion coordination symmetry during the catalytic reaction. Using this new implementation, we identified that the coordination symmetry follows a consistent pattern in the three systems studied, reaching the most symmetrical state near the transition states. We found that the phosphate cleavage proceeds with a concerted bimolecular $\left(\mathrm{A}_{N} \mathrm{D}_{\mathrm{N}}\right)$ mechanism with a loose dissociative transition state, and that it is coupled with a proton transfer step involving a unanimously conserved Asp residue. We show that the main mechanistic effect of the lack of the C-terminal arm is to decouple the phosphate cleavage from the subsequent proton transfer step, resulting in a highbarrier altered reaction pathway.
\end{abstract}


Keywords: QM/MM (quantum mechanics/molecular mechanics), dUTPase, continuous symmetry measure, one-metal ion catalytic mechanism, ab initio, DFT (density functional theory), coupled proton transfer

\section{Introduction}

The dUTPase (deoxyuridine triphosphate nucleotidohydrolase) enzymes catalyze the hydrolysis of dUTP (deoxyuridine triphosphate) to dUMP (deoxyuridine monophosphate) and pyrophosphate to avoid incorporation of uracil into the DNA instead of thymine, and to provide the dUMP precursor for de novo dTTP (deoxythymidine triphosphate) biosynthesis. ${ }^{1}$ An additional enzymatic activity, deamination of dCTP (deoxycytidine triphosphate) to dUTP, may also be carried out by bifunctional dUTPases in Archaea $^{2-5}$ and also in Mycobacterium tuberculosis (M. tuberculosis), which also has a monofunctional dUTPase. ${ }^{6}$ The dUTPases comprise two structurally different classes: ${ }^{7}$ first, the $\beta$ sheet-type dUTPases, possessed by most organisms, employ a one-metal ion catalytic mechanism, ${ }^{8-9}$ and usually form a homotrimer ${ }^{10}$ or a monomer, ${ }^{11}$ while the second class, the $\alpha$ helical-type dUTPases, possessed by kinetoplastids, ${ }^{12}$ form dimers and employ a two-metal ion catalytic mechanism. $^{13-14}$

In $\beta$ sheet-type trimeric dUTPases, each active site is constituted from motifs of all three protomers, which are all required to interact for achieving proper enzymatic activity. ${ }^{15-16}$ These dUTPases have a C-terminal arm structural motif that has sequence similarity to P-loop motifs ${ }^{17}$ also present in triphosphate hydrolysis catalyzing enzymes including kinases, motor proteins, and membrane pumps. ${ }^{18-20}$ In addition to the key C-terminal arm, the requirement of divalent metal ion cofactors for efficient catalysis is also shared by AAA+ (ATPases associated with diverse cellular activities) enzymes in general, commonly a single $\mathrm{Mg}^{2+}$ coordinating the phosphate groups of the substrate. Substitution of this $\mathrm{Mg}^{2+}$ with $\mathrm{Ca}^{2+}$ generally results in substantial loss of activity, ${ }^{21-22}$ with only a few exceptions reported (e.g., for human endogenous retrovirus (HERV-K) dUTPases). ${ }^{23}$ The P loop-like C-terminal arms, the $\mathrm{Mg}^{2+}$ ion bound triphosphate nucleotides, and the binding interface of the remaining two protomers, - all contribute to the resulting three active sites of the native dUTPase complex.

Here, we focus on the role of the C-terminal arm of $M$. tuberculosis dUTPase (Figure 1A) in the catalytic activity and in ligand binding properties. We use both computationally intensive, accurate mixed quantum mechanics/molecular mechanics (QM/MM) calculations ${ }^{24}$ and sitedirected mutagenesis experiments. We study the wild type (WT) dUTPase, a mutant lacking the entire C-terminal arm (denoted by T138Stop), and a second mutant including only a H145A mutation. The C-terminal arm structural motif has several conserved residues (Figure 2 ), one of which, His145, has well-conserved aromatic $\pi-\pi$ interactions with the nucleobase of the substrate. ${ }^{17,25-26}$ His145 has a prominent location in the dUTPase active site: it interacts simultaneously with both the other two protomers and the substrate (Figure 1B). Deletion of the full C-terminal arm results in major activity loss for the T138Stop enzyme. The His to Ala mutation H145A preserves substrate binding; however, the catalytic rate decreases by a factor of 10. The measured binding affinities and catalytic rates of the T138Stop and H145A enzymes are consistent with the essential role of the C-terminal arm in other dUTPase proteins investigated. ${ }^{17,25,27-32}$ 
To better understand the underlying energetic and structural causes determining the catalytic activity, we carried out QM/MM calculations of the reaction mechanism for proton transfer and phosphate cleavage in the WT and the two mutant (H145A and T138Stop) enzyme complexes. We recently identified a one-step associative $A_{N} D_{N}$ catalytic mechanism for the hydrolysis at the $\alpha$-phosphate, involving also a coupled proton transfer step from the nucleophilic water to the catalytic Asp83 residue. ${ }^{33}$ Here we show that essentially the same mechanism takes place in all three dUTPase systems investigated. The coupled proton transfer and the phosphate cleavage occur in a single step that has a loose dissociative transition state (TS) structure. The proton transfer directly follows the phosphate bond breaking, and it is coupled together with the new phosphorous-oxygen bond formation at the TS for the WT. For the H145A and T138Stop enzymes, the proton transfer is decoupled from the phosphorousoxygen bond formation at the TS, in particular for the nearly inactive T138Stop dUTPase. In addition, we also found evidence that the specific metal ion coordination mode may play a prominent role. We analyzed the metal ion coordination during the reaction by calculating a continuous symmetry measure for the $\mathrm{Mg}^{2+}$-ion coordination. ${ }^{34}$ Changes in charge distributions of the three systems for the TS structures also reveal relevant differences in the partial charge transfer from the protein to the substrate due to the missing native proteinprotein and protein-substrate interactions.

Our QM/MM calculations offer unique insights into the structural and electronic changes taking place along the catalytic reaction and, in conjunction with the experimental data, provide evidence on the crucial role of the dUTPase C-terminal arm in modulating ligand binding geometry and catalytic activity. ${ }^{35}$

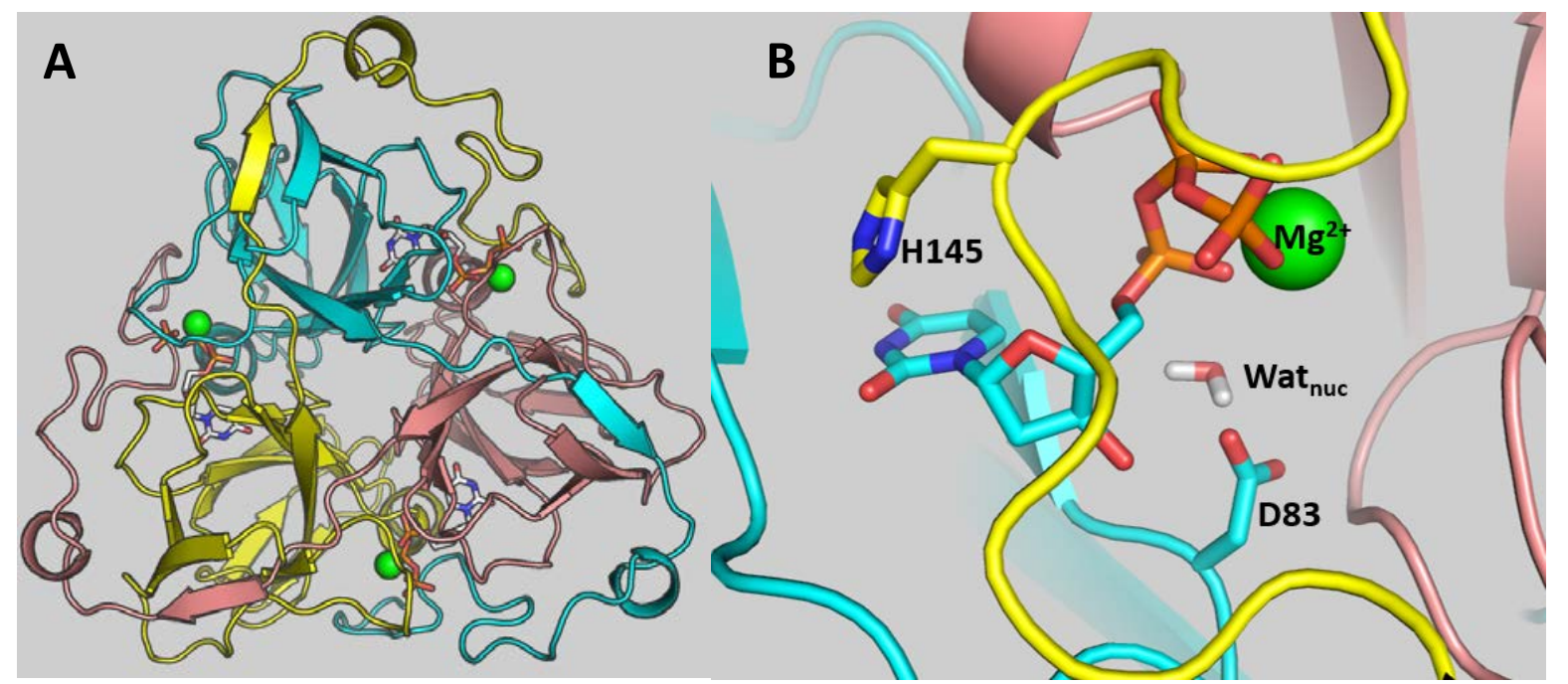

Figure 1. A) Homotrimeric structure of the biological M. tuberculosis dUTPase assembly. Each active site consists of all the three protomers (yellow, cyan, and salmon cartoon), a dUTP molecule (sticks with atomic coloring), and a single $\mathrm{Mg}^{2+}$ ion cofactor (green sphere). B) Active site of $M$. tuberculosis dUTPase from the WT simulation system. H145 (sticks) forms $\pi-\pi$ interactions with the uracil base of the substrate dUTP (sticks) at the interface of the three protomers, interacting with all the three chains (yellow, cyan, and salmon cartoon). 


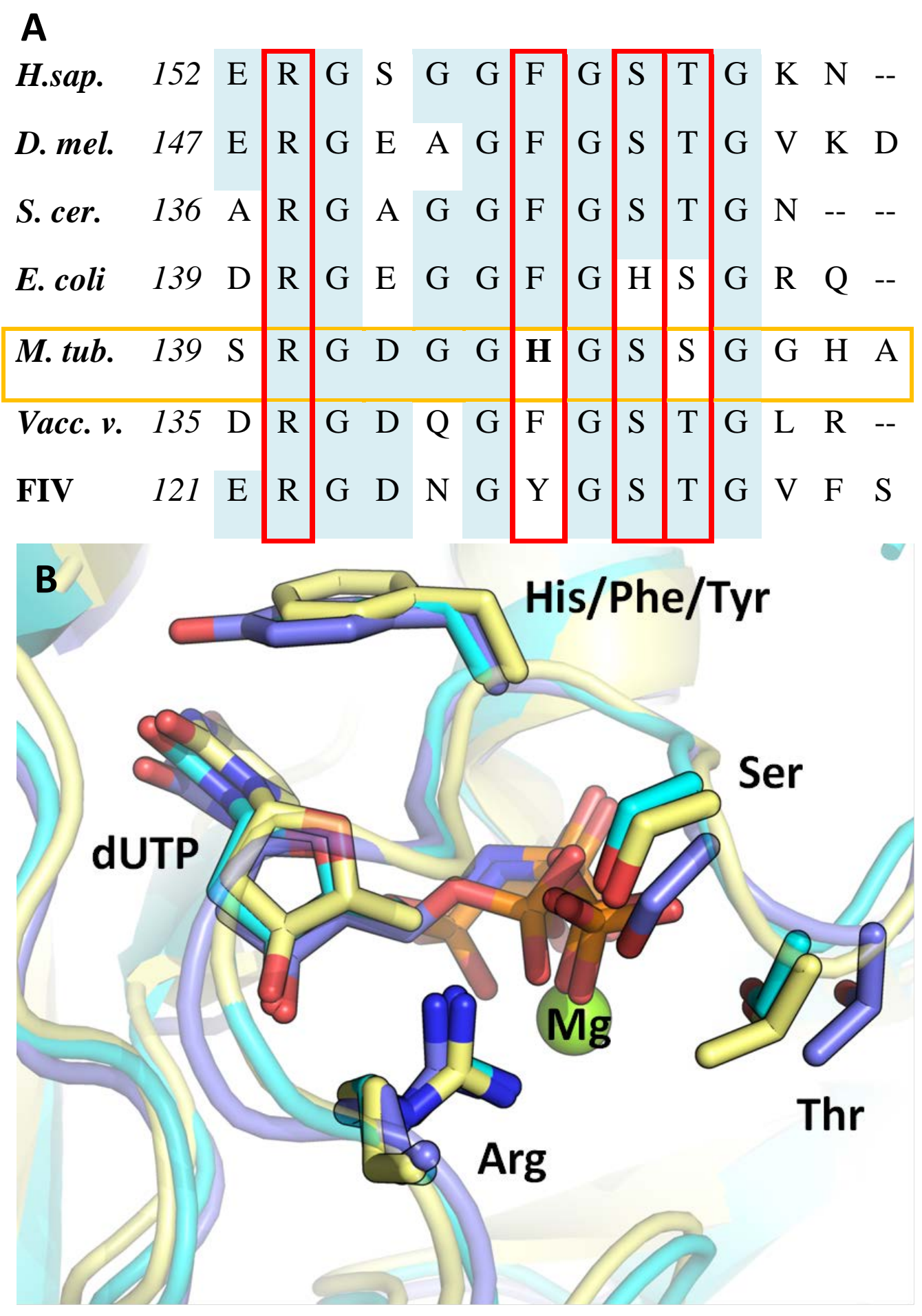

Figure 2. A) Clustal W alignment of the C-termini of dUTPases from widely different species: human (Homo sapiens, $H$. sap.), Drosophila melanogaster (D. mel.), Saccharomyces cerevisiae (S. cer.), E. coli, M. tuberculosis, Vaccinia virus (Vacc. v.), and feline immunodeficiency virus (FIV). The conserved residues coordinating the dUTP are shown within a red frame. B) Superimposed structures of the active sites of human, mycobacterial and FIV dUTPases (PDB IDs: 3EHW (unpublished), 2 PY4 ${ }^{26}$ and 1F7R, ${ }^{36}$ respectively). The conserved residues coordinating the dUPNPP molecule, which is a slowly hydrolyzable dUTP analog, are shown as sticks, protein shown as cartoon (yellow, cyan and dark blue, respectively). Note that the presented FIV dUTPase 
structure contains dUDP with trans conformation instead of dUPNPP and there is no $\mathrm{Mg}^{2+}$ ion coordinated to the phosphate chain of the substrate analog.

\section{Materials and Methods}

\section{Experimental Methods.}

Reagents. Molecular biology products were obtained from New England Biolabs (US) and Fermentas (Canada), electrophoresis and chromatography reagents were from Bio-Rad (US) and Qiagen (Netherland). Phenol red was from Merck (Germany), dUDP and dUPNPP ( $\alpha, \beta-$ imido-dUTP) was from Jena Bioscience (Germany); dUTP and other chemicals were from Sigma-Aldrich (US).

Mutations on the C-terminal arm. C-terminal arm mutants have been generated to elucidate the roles of protein-protein and protein-substrate interactions of this conserved arm motif on dUTPase function. Two mutants were generated (H145A and T138Stop) focusing on the conserved residue of His145 (Figures 1 and 2), and a mutant lacking the last 17 residues of the C-terminal arm. Site-directed mutagenesis was performed by Stratagene's QuikChange method and was verified by sequencing. Mutagen forward and reverse primers were the following:

H145A

$$
\begin{aligned}
& \text { 5'-GGCGACGGTGGCGCGGGTTCCTCCGGC-3' } \\
& \text { 5'-GCCGGAGGAACCCGCGCCACCGTCGCC-3' } \\
& \text { 5'-GGGCTGGCCTCGTGATCCCGCGGCGACGG-3' } \\
& \text { 5'-CCGTCGCCGCGGGATCACGAGGCCAGCCC-3' }
\end{aligned}
$$

T138Stop

Cloning, protein expression and purification were performed as described previously in Varga et al. ${ }^{26}$ and as follows. Recombinant dUTPase with an N-terminal 6xHis-tag was expressed in E. coli strain BL21(DE3)pLysS. The final supernatant after cell extraction was loaded on a Ni-NTA column (Novagen) and purified according to the Novagen protocol. Histag purification allows for specific purification of the recombinant proteins from the endogenous one. Protein concentration was measured using the Bradford method (Bio-Rad Protein Assay) and is given in monomers. Proteins were dialyzed against a buffer $\mathrm{pH} 7.5$, containing $20 \mathrm{mM}$ HEPES, $100 \mathrm{mM} \mathrm{NaCl}, 2 \mathrm{mM} \mathrm{MgCl} 2$ and $1 \mathrm{mM}$ DTT.

Steady-state colorimetric dUTPase assay was performed as described in Pecsi et al. ${ }^{25}$ Phenol red indicator assay was used to detect protons released in the dUTPase reaction. 0.1-2 $\mu \mathrm{M}$ protein in a buffer $\mathrm{pH} 7.5$ was used containing $1 \mathrm{mM}$ HEPES, $100 \mathrm{mM} \mathrm{KCl}, 40 \mu \mathrm{M}$ phenol red and $5 \mathrm{mM} \mathrm{MgCl}$. A Specord 200 (Analytic Jena, Germany) spectrophotometer and 10 -mm path length thermostated cuvettes were used at $20^{\circ} \mathrm{C}$ and absorbance was recorded at $559 \mathrm{~nm}$. Initial velocity was determined from the first $10 \%$ of the progress curve. The Michaelis-Menten equation was fitted to the steady-state curves.

Enzyme-ligand dissociation constant measurements were carried out as circular dichroism intensity titrations. CD spectra were recorded at $20^{\circ} \mathrm{C}$ on a JASCO 720 spectropolarimeter using a $10 \mathrm{~mm}$ path length cuvette. Protein concentration was $50 \mu \mathrm{M}$ in a buffer $\mathrm{pH} 7.5$, containing $20 \mathrm{mM}$ HEPES, $50 \mathrm{mM} \mathrm{NaCl}$ and $2 \mathrm{mM} \mathrm{MgCl}_{2}$. A spectrum between 250 and 290 $\mathrm{nm}$ was recorded at each nucleotide concentration as described in Pecsi et al. ${ }^{25}$ Differential curves were obtained by subtracting the signal of the ligand alone from that of the corresponding complex. Differential ellipticity at $269 \mathrm{~nm}$ was plotted against the ligand concentration to obtain the binding curves. 
Protein solution was titrated with dUPNPP or dUDP using minute aliquots from a concentrated stock solution. Titration data were fit to the equation describing 1:1 stoichiometry for the dissociation equilibrium with no cooperativity:

$$
y=s+\frac{A\left[(c+x+K)-\sqrt{(c+x+K)^{2}-4 c x}\right]}{2 c}
$$

Where, $x$ is the nucleotide concentration and $y$ is the circular dichroism intensity, $s=y$ at $x=0$, $\mathrm{A}$ is the amplitude of the circular dichroism intensity change, $c$ is the enzyme concentration and $K$ is the dissociation constant $\left(\mathrm{K}_{\mathrm{d}}\right)$.

Thermal stability measurements were carried out with a JASCO 720 spectropolarimeter using a $1 \mathrm{~mm}$ path length cuvette. $25 \mu \mathrm{M}$ enzyme was measured in a buffer $\mathrm{pH} 7.5$, containing $100 \mathrm{mM} \mathrm{NaH}_{2} \mathrm{PO}_{4}$ and $2 \mathrm{mM} \mathrm{MgCl}_{2}$. The solution was heated from 20 to $80{ }^{\circ} \mathrm{C}$ and differential ellipticity was recorded by every $0.5^{\circ} \mathrm{C}$ at $210 \mathrm{~nm}$. The obtained values were plotted against temperature and the following Boltzmann curve was fitted:

$y=A 2+\frac{A 1-A 2}{1+e^{\frac{x-x 0}{d x}}}$

where $A 1$ is the initial value and $A 2$ is the final value of the differential ellipticity, $\mathrm{dx}$ is the time constant and $x 0$ is the inflection point of the curve which is the melting point.

Data fitting and statistical analysis. All experimental measurements were carried out at least three times. Error bars represent standard deviations of the mean of the measurements. Data fitting was performed using Origin 7.5 (OriginLab Corp., Northampton, MA).

\section{Computational Methods.}

Structural Model. The calculations were initiated from the X-ray crystal structures of the wild type and mutant $M$. tuberculosis dUTPase enzymes (Table S1). The original dUPNPP ligand in the crystal structures was manually modified to dUTP. There is a lack of the catalytic water in the T138Stop crystal structure, however a distinct electron density with a high temperature factor can be seen at the usual position of the attacking water molecule and this information was used to position a catalytic water molecule into the structure of the T138Stop mutant (Figure S2B). Coordinates of the hydrogen atoms were generated with $\mathrm{CHARMM}^{37}$ using standard protonation states of all ionizable residues (also see SI text about potential protonation states of H145). Crystallographic water molecules in the vicinity of the protein and the substrate were preserved while the system has been placed in a cube of TIP3P water to cover at least $10 \AA$ radius in all directions from the protein. Several water molecules were then replaced with a Monte Carlo procedure by ions to account for a $0.10 \mathrm{M} \mathrm{NaCl}$ solution, and further $\mathrm{Na}^{+}$ions were added for neutralization. ${ }^{38}$ CHARMM27 extended atom force field was used for the molecular mechanics interactions, with periodic boundary conditions, Ewald summation and a $12 \AA$ cutoff for the evaluation of the non-bonded interactions. After an initial equilibration for $10 \mathrm{~ns}$, with constraints to the initial PDB structure, the final structure was taken as the starting point for subsequent MM and QM/MM minimizations. 


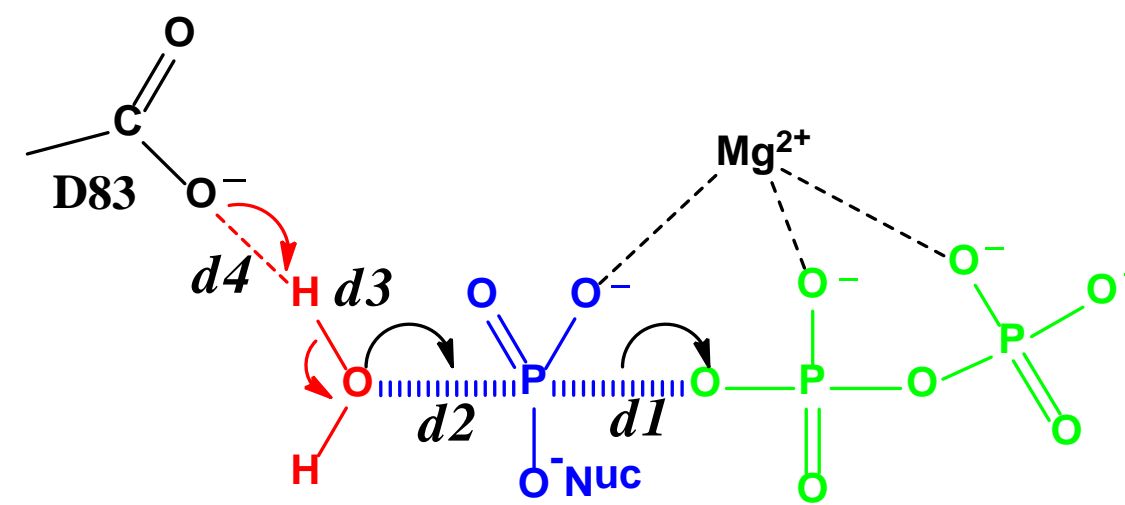

Figure 3. Schematic representation of the reaction coordinate used in the $Q M / M M$ simulations. We defined $Q=Q_{E T}+0.5 Q_{P T}$, where $Q_{E T}=d 1-d 2$ denotes the difference between the bond breaking (d1) and bond forming (d2) distances for the phosphate cleavage (generalized electron transfer coordinate), and $Q_{P T}=d 3-d 4$ is the difference between the bond breaking $(d 3)$ and forming $(d 4)$ distances for the proton transfer from the water to the Asp83.

QM/MM setup and minimizations. The equilibrated classical system was trimmed to a sphere of $21 \AA$ radius centered at the position of the $\mathrm{P}_{\alpha}$ atom of the dUTP, and all residues further than $15 \AA$ were kept fixed. Q-Chem program ${ }^{39}$ version 3.1 was used to perform QM calculations, at the B3LYP ${ }^{40} / 6-31+G(d)$ density functional theory (DFT) level of theory. The quantum mechanical system was coupled with the CHARMM program ${ }^{37}$ using full electrostatic embedding. ${ }^{41}$ Standard link atom treatment was used to bisect bonds between the $\mathrm{QM}$ and $\mathrm{MM}$ region, adding hydrogen atoms for the missing ligands. For the $\mathrm{QM} / \mathrm{MM}$ minimizations, a complex reaction coordinate $(Q$, Figure 3$)$ was defined as the difference between the forming and breaking bond distances in which both phosphate transfer (ET, in analogy with generalized electron transfer) and proton transfer (PT) are incorporated. ${ }^{42-43}$ Using this reaction coordinate, the geometries were minimized starting with the initial enzyme-substrate structures and following the reaction through the transition state to the product state. Minimizations were performed using the adopted basis Newton-Raphson scheme as implemented by D. J. States ${ }^{44}$ in CHARMM, both in the forward and backward directions to obtain hysteresis-free results. ${ }^{42}$

The quantum region contained the Thr81 and Ile82 backbones, the amide group of Gln113, the side chains of Asp83, Ser65, Thr69 (only in the T138Stop mutant), Ser147 and Ser148 (in the WT and H145A mutant), the side chain of residue 145 (His in the WT, Ala in the H145A mutant), the guanidino group of Arg140 (in the WT and H145A mutant) and Arg64 (only in the T138Stop mutant), the $\mathrm{Mg}^{2+}$ ion with its full coordination shell, nearby crystallographic water molecules, and the dUTP molecule (Figure S1). Thus, the WT, H145A, and T138Stop systems contained 132, 137, and 108 atoms, respectively, in the minimizations with the largest QM region.

After the full QM/MM minimization of the corresponding structures, the charge distribution was analyzed with the Gaussian program package ${ }^{45}$ using the CHELPG charge evaluation ${ }^{46}$ based on fitting the atomic charges to reproduce the electrostatic potential. ${ }^{47}$ In these calculations the same QM region was used at the same level of theory with an identical polarization scheme as done in the QM/MM minimizations, including the same protein environment in the calculations as external point charges. 
QM/MM dynamics. Short downhill QM/MM dynamics trajectories were generated starting from the minimized WT system near the transition state. The quantum region contained the Thr81 and Ile82 backbones, the amide group of Gln113, the side chains of Asp83, Ser65, His145, Ser147, and Ser148, the guanidino group of Arg140 the $\mathrm{Mg}^{2+}$ ion with its full coordination shell, nearby crystallographic water molecules, and the dUTP molecule (121 atoms in total, Figure S1A). We used Langevin dynamics with a friction coefficient of $8 \mathrm{ps}^{-1},{ }^{48}$ and an integration time step of $1 \mathrm{fs}$. During the trajectories, the outer simulation shell was kept fixed as described above. Keeping also the QM region fixed initially, 10 ps long classical MD simulations were carried out first for each downhill trajectory to obtain an ensemble of TS structures with respect to the classical region. These simulations were then followed by unconstrained dynamics using QM/MM calculations with no atoms fixed except for residues further than $15 \AA$ from the $\mathrm{P}_{\alpha}$ atom.

Downhill trajectories were also generated for the T138Stop mutant as described above. The quantum region contained the Thr81 and Ile82 backbones, the amide group of Gln113, the side chains of Asp83, Ser65, Thr69, the $\mathrm{Mg}^{2+}$ ion with its full coordination shell, nearby crystallographic water molecules, and the dUTP molecule (95 atoms in total, Figure S1C).

Symmetry measure calculations. To analyze the coordination shell of the $\mathrm{Mg}^{2+}$ ion, we introduce a continuous, dimensionless symmetry parameter $S$, similarly to the parameter introduced in Zabrodsky et al. ${ }^{34}$ for general geometric structures. Our symmetry measure $(S)$ is defined as

$$
S=\min _{\{r, \mathbf{M}\}}\left(\frac{1}{6} \sum_{i=1}^{6}\left|\mathbf{e}_{i}-\mathbf{M}\left(\frac{\mathbf{x}_{i}}{r}\right)\right|^{2}\right) .
$$

Here, $\mathbf{x}_{i}$ are the oxygen position vectors $(i=1, \ldots, 6)$ for a given structure, and $\mathbf{e}_{i}$ are the 6 unit vectors corresponding to the ideal symmetrical octahedral geometry $\left(\mathbf{e}_{1}=(1,0,0), \mathbf{e}_{2}=(0,1,0)\right.$, $\mathbf{e}_{3}=(0,0,1), \mathbf{e}_{4}=(-1,0,0), \mathbf{e}_{5}=(0,-1,0)$, and $\left.\mathbf{e}_{6}=(0,0,-1)\right)$. For each optimized structure along the reaction profile, we determined $S$ as the minimum distance to an ideal octahedron. This was done by simultaneous optimization of four parameters: (i) the scaling distance $r$, and (ii) the three Euler angles corresponding to the independent elements of the 3D rotation matrix, $\mathbf{M}$. For each optimized structure along the path, we minimized the average distance between the ideal fully symmetric octahedron $\left(\mathbf{e}_{i}\right)$ and the rotated, distance scaled, adimensional/dimensionless, octahedron with edges composed of the actual oxygen atom positions of the $\mathrm{Mg}^{2+}$ ligands. 


\section{Results and Discussion}

Steady-state activities of the WT, T138Stop and H145A mutants were determined by fitting Michaelis-Menten curves to the measured data points (Figure 4). The activity of the H145A mutant decreased by one order of magnitude, while the T138Stop mutant shows three orders of magnitude activity decrease. Both values are in agreement with the homologous mutations in the human dUTPase enzyme. ${ }^{17,25}$

A

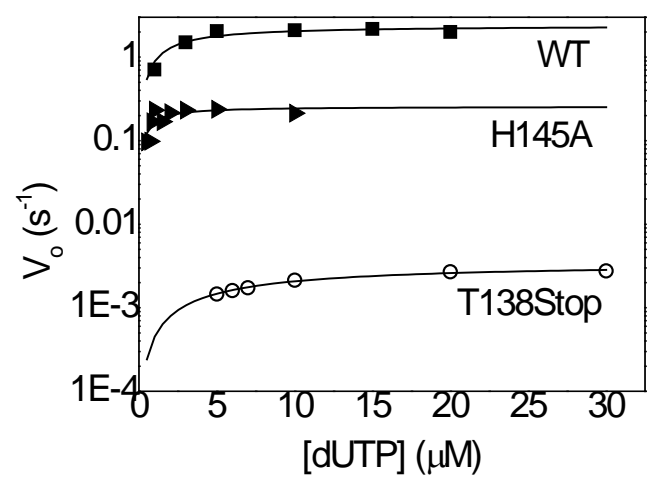

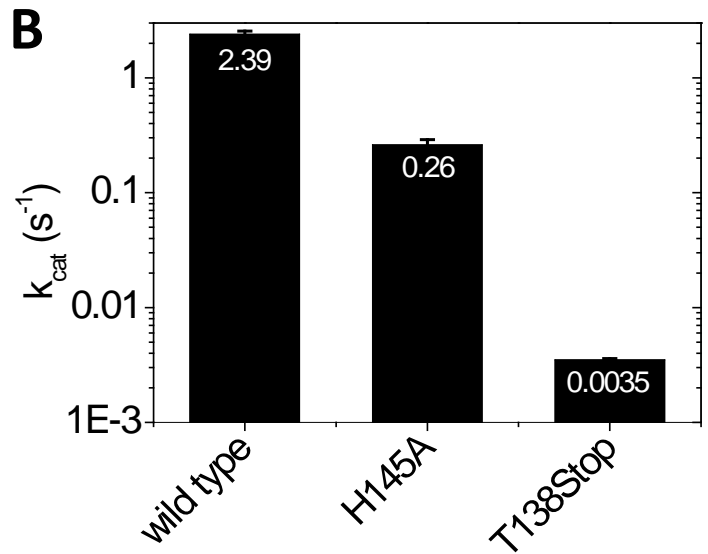

Figure 4. A) Michaelis-Menten enzymatic activity curves of the wild type and the mutants. B) Steady-state activity of the dUTPase mutants. Note that both the initial velocity $\left(\mathrm{V}_{0}\right)$ and the catalytic rate constant $\left(\mathrm{k}_{\mathrm{cat}}\right)$ values of the mutants are shown on a logarithmic scale.

Table 1.Measured kinetic and ligand binding parameters of the mutants.

\begin{tabular}{|l|c|c|c|}
\hline Mutant & WT & H145A & T138Stop \\
\hline $\mathbf{K}_{\mathbf{c a t}}\left(\mathbf{s}^{-\mathbf{1}}\right)$ & $3.1 \pm 0.06$ & $0.26 \pm 0.03$ & $0.0035 \pm 0.0001$ \\
\hline $\mathbf{K}_{\mathbf{M}}(\boldsymbol{\mu M})$ & $0.46 \pm 0.2$ & $0.6 \pm 0.2$ & $6.7 \pm 0.4$ \\
\hline $\mathbf{K}_{\mathbf{d ~ d U P N P P}}(\boldsymbol{\mu M})$ & $0.9 \pm 0.5$ & $2.1 \pm 1.0$ & $3.9 \pm 1.3$ \\
\hline $\mathbf{K}_{\mathbf{d ~ d U D P}}(\boldsymbol{\mu M})$ & $12.7 \pm 3.6$ & $13.6 \pm 2.9$ & $13.7 \pm 2.3$ \\
\hline $\left.\boldsymbol{\Gamma}_{\mathbf{m}} \mathbf{(}^{\mathbf{0}} \mathbf{C}\right)$ & $61.2 \pm 0.1$ & $58.0 \pm 0.4$ & $62.4 \pm 0.1$ \\
\hline
\end{tabular}

Ligand binding experiments show that the mutants bind the dUTP analog $\alpha, \beta$-imido-dUTP (dUPNPP) only 2-4 times weaker than it does the wild type (Table 1). This small change in ligand binding is consistent with the observed structural similarity of the H145A and T138Stop mutants compared to the wild type (see below). We also measured the dUDP binding in the mutant proteins, and found no difference compared to the wild type. Since the mutations had some effect on the binding of dUPNPP, but not on the binding of dUDP, the mutated residues thus may promote the selectivity between dUTP and dUDP as described previously. $^{17}$ 
No large scale structural changes. Thermal stability of the mutants and the wild type has been measured to ensure that the observed differences are not the results of changes in the entire enzyme structure. The measured melting temperatures are similar for both the mutants and the wild type, confirming that the mutations did not induce significant changes in the global protein structure (Table 1).

Crystal structures were previously obtained for the $\mathrm{H} 145 \mathrm{~A}^{25}$ and for the T138Stop ${ }^{15}$ mutants. These structures demonstrated that the substrate binding is not significantly affected by the mutations, the overall protein assemblies and substrate binding remained largely similar. The RMSD of the common amino acid atoms with respect to the wild type is $0.12 \AA$ for H145A and $0.20 \AA$ for T138Stop mutant. A smaller, but potentially important change in the substrate position was observed for the T138Stop mutant. The substrate gamma phosphate orientation changed compared to the coordinating $\mathrm{Mg}^{2+}$ that is not observed for the H145A (Figure S2A). All other residues remained within $0.5 \AA$ compared to the wild type for both the T138Stop and the H145A mutants.

Structural changes compared to the crystal structures. There are no significant changes between our structures obtained after minimization and the original crystallographic structures. The overall RMSD of the optimized reactant state (RS) structures and the crystallographic structures are $0.252 \AA$ for the WT, $0.477 \AA$ for the H145A, and $0.336 \AA$ for the T138Stop mutant. The largest deviations are $1.77 \AA$ for the Asp28 $\mathrm{O}$ in the case of the H145A mutant and $1.37 \AA$ for the Ser65 O in the case of the T138Stop mutant. These changes are relatively small compared to the crystal structure resolution (1.80 and $1.25 \AA$ for the H145A and T138Stop mutant, respectively), and may also be affected by the presence of the dUPNPP instead of the native dUTP substrate. It is worthwhile to note that both the Asp28 and Ser65 residues are well conserved and found to be important for efficient catalysis. ${ }^{15,49}$ An analogous residue to Ser65 has also been found in an alternative conformation in Bacillus subtilis dUTPase. ${ }^{50}$ 


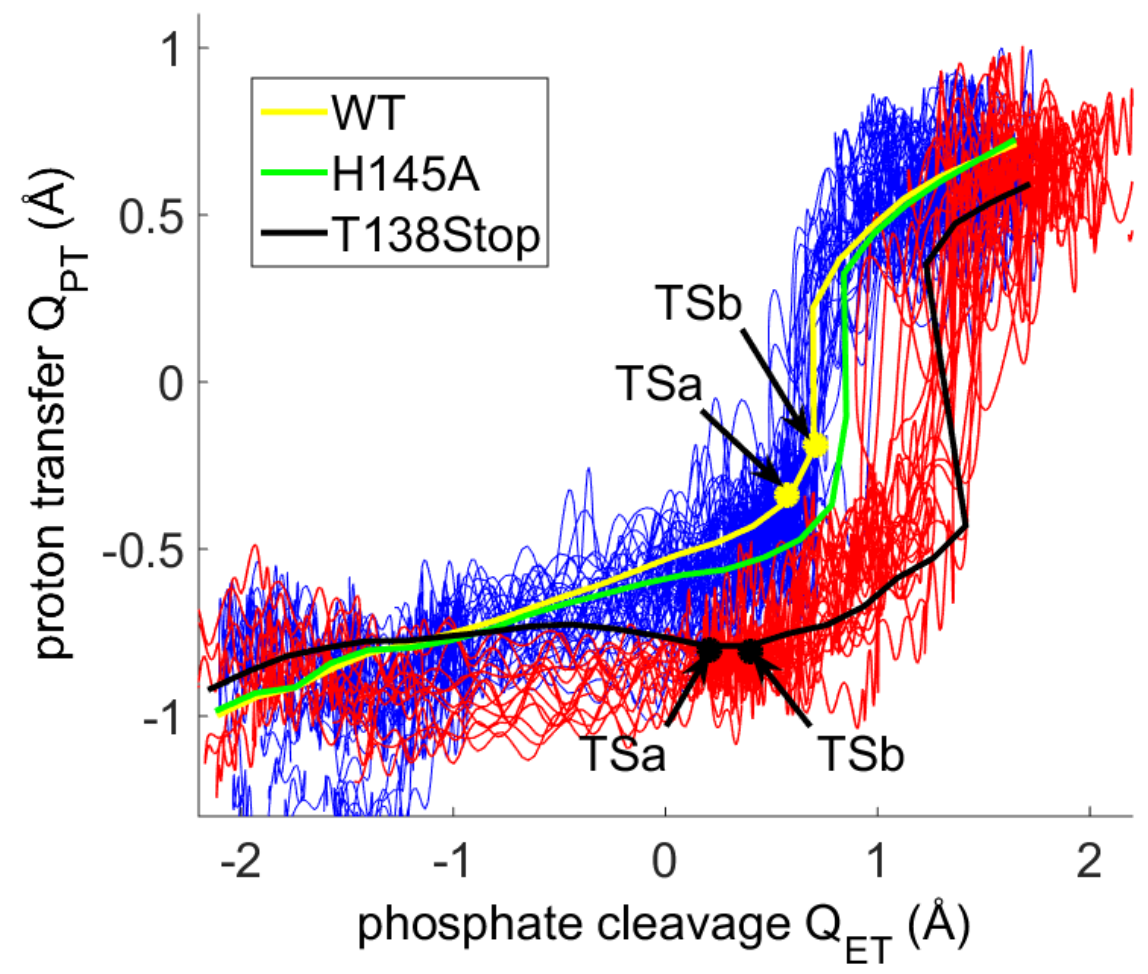

Figure 5. Reaction pathways along the $\mathrm{Q}_{\mathrm{PT}}$ and $\mathrm{Q}_{\mathrm{ET}}$ reaction coordinates. Minimum energy pathways are shown for the WT (yellow), H145A (green) and T138Stop (black) systems. Unbiased downhill QM/MM MD trajectories are also shown for the WT (blue) enzyme and for the T138Stop (red) mutant. The downhill trajectories (thin lines) were initiated from near the corresponding transition state (TS) positions for each system (arrows and symbols). TSa and TSb correspond to the nearest points to the transition state on the reactant and on the product side along the minimized pathways, respectively. (See the "Downhill trajectories" section for further details.)

Reaction mechanism. To investigate the detailed reaction mechanism and the effects of the mutations on the catalytic activity, we performed hybrid QM/MM calculations on the wild type, H145A and T138Stop mutants. Our preliminary minimizations, in which only the phosphate bond forming and breaking coordinates were constrained, confirmed that the proton transfers from the nucleophilic water molecule to the oxygen of the Asp83 carboxylic group. We observed that, when no bias was used on the proton transfer process (i.e., only the $Q_{E T}$ coordinate is biased), as the product was approached, the proton spontaneously transferred to the Asp83. Asp83 is a strictly conserved residue, essential for catalytic activity of trimeric dUTPases. Accordingly, it has been identified in numerous dUTPases as being the universal proton acceptor in the catalytic mechanism. ${ }^{10,23,51-52}$ We therefore performed subsequent minimizations along the reaction pathway using the complete reaction coordinate, defined as $Q=d 1-d 2+0.5(d 3-d 4)$ (Figures 3 and 5). $Q$ accounts for all bond breaking and forming processes involved in the rate limiting catalytic step, including the proton transfer from the nucleophilic water molecule to the acceptor Asp83 residue. For all three obtained pathways the reaction follows a one-step $A_{N} D_{N}$ mechanism regarding the phosphate hydrolysis (Figure S3). The proton transfer from the nucleophilic water to the Asp83 immediately follows the phosphorous bond forming and bond breaking steps in a concerted mechanism (Figure 5). The reaction is initiated by the attack of the nucleophilic water, leading to an associative pathway for the phosphate cleavage that has been identified in 
solution and in several enzymes that carry out phosphate cleavage or transfer (see e.g., Florián et al. ${ }^{53-54}$ and Klahn et al. ${ }^{55}$ ).

This mechanism is also consistent with the experimentally observed $\mathrm{pH}$ change that is used to directly follow the reaction kinetics, ${ }^{56}$ and with the lack of observed intermediates for the phosphate hydrolysis. ${ }^{33}$ It is found in E. coli dUTPase that two protons transfer to the reaction medium in a concerted mode, immediately following the rate limiting step. ${ }^{56}$ These proton transfer events were also found dependent on two ionizable groups in the protein substrate complex with $\mathrm{p} K_{\mathrm{a}}$ values of 5.8 and $10.3 .{ }^{56}$ While the WT and H145A reaction pathways are closely similar to each other, with the H145A pathway being somewhat less concerted (Figures 5 and 6B), a striking difference is observed for the T138Stop mutant. For this enzyme, which lacks the C-terminal arm, we observed a much more stepwise reaction profile (Figures 5 and 6), with the proton transfer being decoupled from the phosphate cleavage. A comparison of the bond forming and breaking distances (Figure 6B) reveals that the crossing points of equal P-O distances happen the earliest for the T138Stop mutant, followed by the H145A and the WT enzymes along the reaction coordinate $Q$. Accordingly, the crossing point for the $\mathrm{O}-\mathrm{H}$ distances is located along Q first for the WT enzyme, followed closely by the H145A, and finally it takes place at the largest Q value for the T138Stop system. We find consistently that the extent of concertedness of the proton transfer (PT) correlates with the calculated energy barrier, and the experimental activity (cf. also Tables 1, 2).

Table 2. Measured activation free energies and calculated activation energies (kcal/mol).

\begin{tabular}{lcc}
\hline & measured $\Delta \mathrm{G}^{\ddagger}$ & calculated $\Delta \mathrm{E}^{\ddagger}$ \\
\hline wild type & 16.6 & 18.0 \\
H145A & 17.9 & 22.6 \\
T138Stop & 20.4 & 24.4 \\
\hline
\end{tabular}




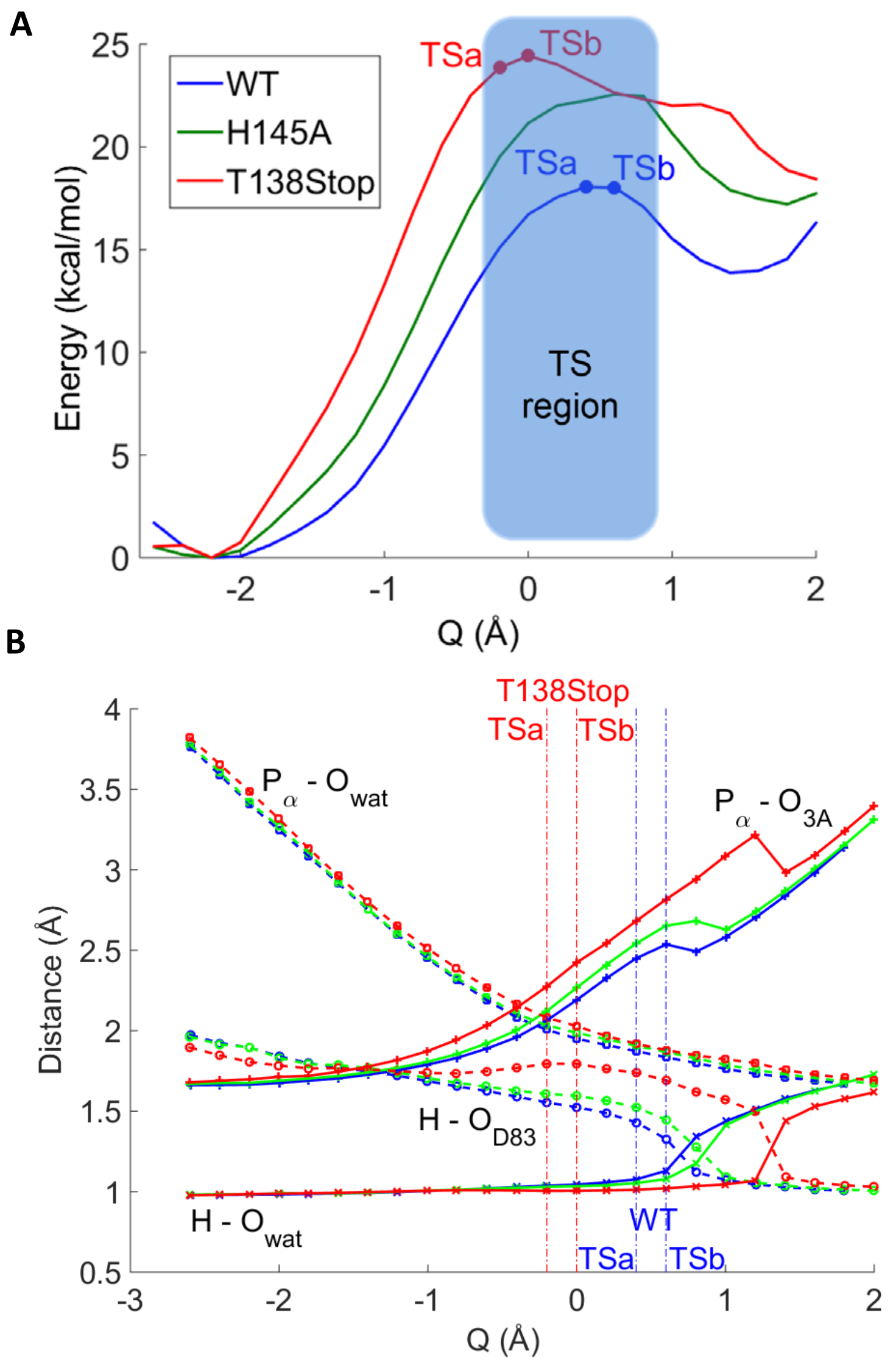

Figure 6. A) Energy profiles for the catalytic reaction and B) distances of the forming $\left(P_{\alpha}-O_{\text {wat, }}\right.$, dashed lines and squares), breaking $\left(\mathbf{P}_{\alpha}-\mathbf{O}_{3 \mathrm{~A}}\right.$, solid lines and + symbols), forming $\mathrm{H}-\mathrm{O}_{\mathrm{D} 83}$ (dashed lines and circles), and breaking open $\mathrm{H}-\mathrm{O}_{\text {wat }}$ (solid lines and 
x symbols) bonds along the reaction coordinate $Q$. WT, H145A and T138Stop systems are shown with blue, green and red, respectively. TS region is highlighted for WT and T138Stop. TSa and TSb (vertical dash-dot lines) correspond to adjacent points at the TS along the optimized pathways, at the reactant and product side, respectively. (See the "Downhill trajectories" section for further details.)

The reaction energies (Table 2 and Figure 6A) are in very good qualitative agreement with the experimental catalytic rates, particularly the observed trend of decreased catalytic activities of T138Stop with respect to WT and H145A. The calculated activation energies are shown in Table 2, together with the activation Gibbs free energies which were obtained from the measured catalytic rate constants using a $6.1 \times 10^{12} \mathrm{sec}^{-1}$ prefactor. The energy barrier for the WT enzyme is in excellent quantitative agreement with the derived experimental activation free energy. The order of activities among the three enzymes is also properly predicted. The calculated barriers are higher for the H145A and T138Stop mutants than the experimentally observed ones, however their relative activities are recovered consistently. Future, more computationally demanding free energy calculations with extensive sampling could be used to account quantitatively for the energy profiles evidenced in this study.

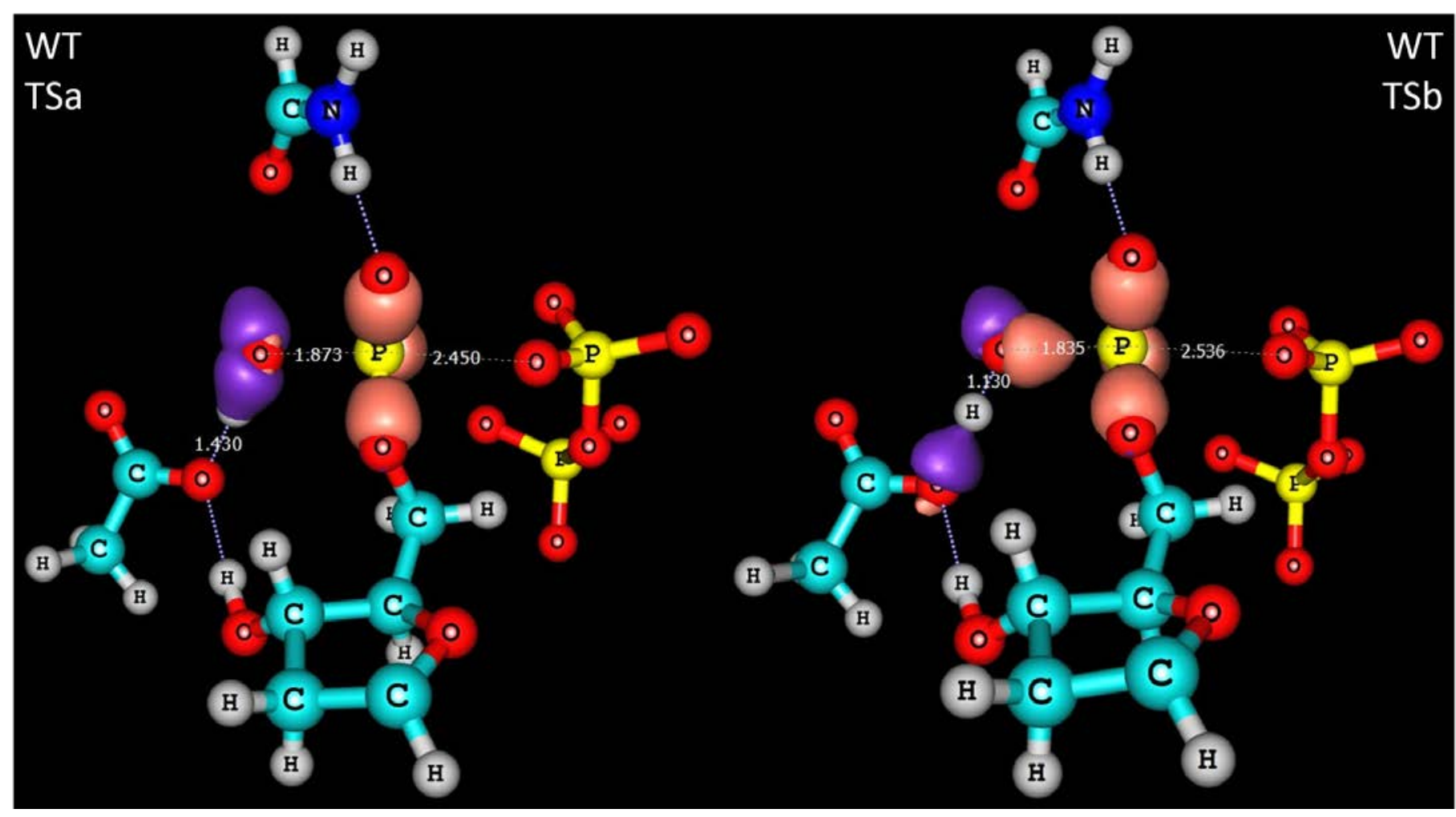




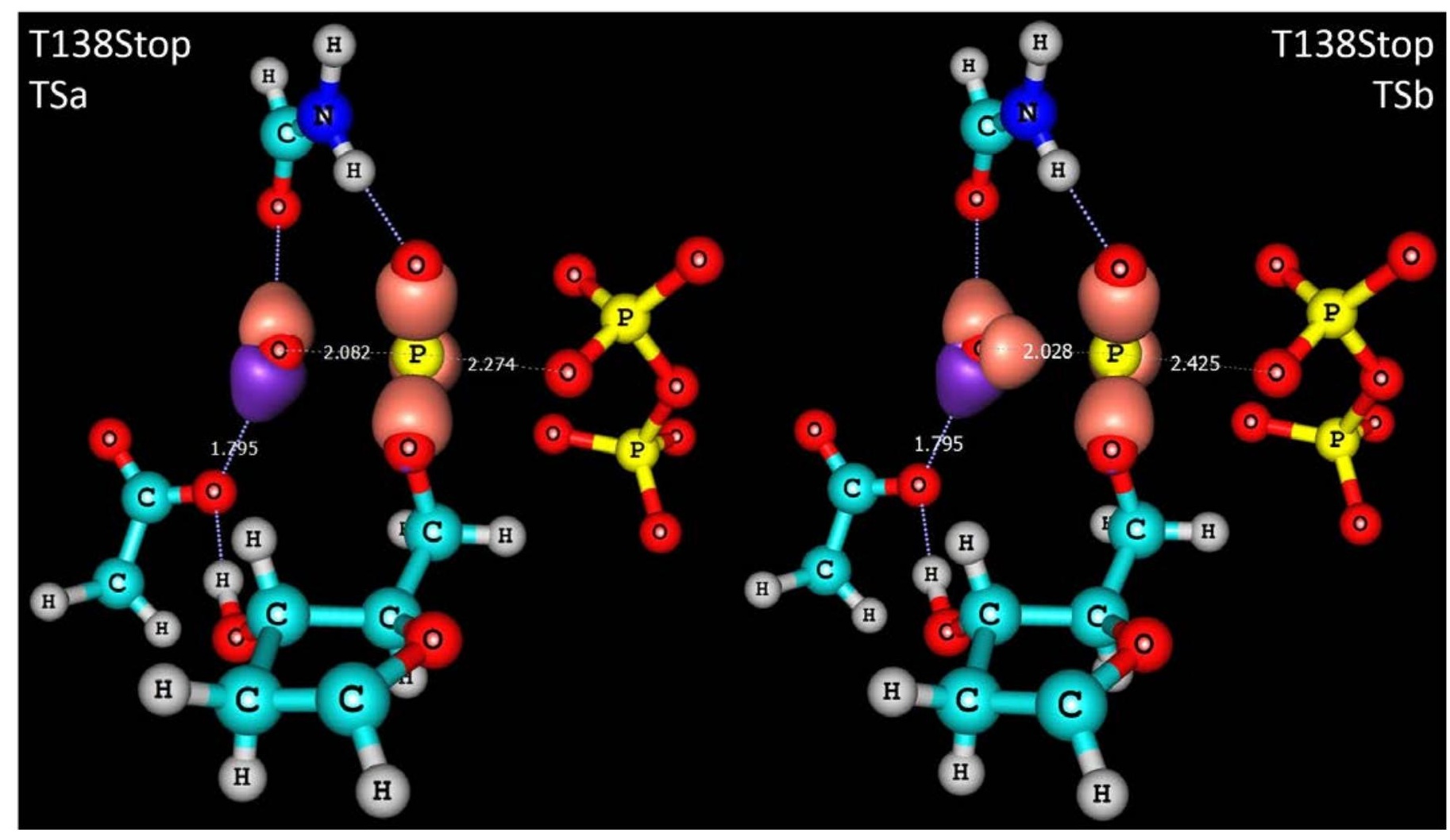

Figure 7. NBO analysis of the transition state structures in the WT and T138Stop enzymes. The molecular orbitals at the 0.2 au electron density level are shown for all natural bonds of only the $H_{\text {wat }}$, $\mathbf{O}_{\text {wat }}$ and $\mathbf{P}_{\alpha}$ atoms. For clarity, only part of the $Q M$ region corresponding to the deoxyribose triphosphate, the nucleophilic water, the proton accepting Asp83 sidechain and the Thr81 and Ile82 backbones are shown.

Transition states geometries and electronic structure. To characterize the transition states, we have analyzed the geometrical parameters corresponding to the bonds breaking and forming during the phosphate cleavage and the proton transfer processes (Table S2). In all three systems the transition state corresponds to long $\mathrm{P}_{\alpha}-\mathrm{O}_{3 \mathrm{~A}}$ leaving group distances, viz. in the range of 2.3-2.6 $\AA$, where the $\mathrm{P}_{\alpha}-\mathrm{O}_{3 \mathrm{~A}}$ bond is already broken. These late transition state structures thus have a dissociative character, and fit well into the loose transition states extensively described in previous experimental studies, based on oxygen kinetic isotope effect data and the slopes of Brønsted plots in linear free energy relationships for analogous phosphate cleavage reactions in enzymes and in solution. ${ }^{57-60}$ Interestingly, the $\mathrm{O}_{\text {wat }}-\mathrm{P}_{\alpha}$ bond length shows greater variations at the TS for the three enzymes; it is the shortest for the WT, and the longest for the T138Stop mutant. Overall, the WT enzyme presents the most "loose" transition state geometry, whereas the almost inactive T138Stop enzyme has the most compact transition state.

To further compare the bonding patterns at the transition states of the three different systems, we also performed Natural Bond Orbital (NBO) analysis at the respective transition state geometries. Consistent with the coupled proton transfer pathway (Figure 5), the bonding analysis reveals simultaneous occurrence of the following events: bond forming between the $\mathrm{O}_{\text {wat }}-\mathrm{P}_{\alpha}$ atoms, bond breaking at the $\mathrm{O}_{\text {wat }}-\mathrm{H}_{\text {wat }}$ bond, and formation of the new $\mathrm{O}_{\mathrm{D} 83}-\mathrm{H}_{\text {wat }}$ bond (Figure 7, top). The two structures at the barrier top correspond to the changing bonding pattern with respect to these three bonds, highlighting all the bonds formed by the $\mathrm{H}_{\text {wat, }}, \mathrm{O}_{\text {wat }}$, 
and $\mathrm{P}_{\alpha}$ atoms in each case. The H145A enzyme has a more flat transition state region, with already formed $\mathrm{O}_{\text {wat }}-\mathrm{P}_{\alpha}$ bond at the $Q=0.2 \AA$ and $Q=0.8 \AA$ regions (Figure S4). The energy then finally drops corresponding to the deprotonation of the water at larger reaction coordinate values (Figure 6A). The proton transfer and the phosphate cleavage are the most decoupled for the T138Stop enzyme, where the transition state corresponds only to the formation of the $\mathrm{O}_{\text {wat }}-\mathrm{P}_{\alpha}$ bond (Figure 7, bottom). The NBO analysis provides a consistent picture clarifying the nature of the chemical bonding at the transition states in all three systems. We find that the $\mathrm{P}_{\alpha}-\mathrm{O}_{3 \mathrm{~A}}$ bond between the leaving group and the phosphorous is broken in all three cases, presenting a concerted mechanism with a loose dissociative TS. The NBO analysis also confirms the simultaneous bond formation of the new $\mathrm{O}_{\text {wat }}-\mathrm{P}_{\alpha}$ bond and the proton transfer from the water to the Asp83 for the WT system, whereas the transition state corresponds to the formation of the new $\mathrm{O}_{\text {wat }}-\mathrm{P}_{\alpha}$ bond only in the T138Stop enzyme, and the proton transfer follows subsequently.

Solvent kinetic isotope effects. To determine the involvement of the proton transfer in the catalytic reactions, we carried out frequency calculations at the reactant and transition states using the $\mathrm{QM} / \mathrm{MM}$ optimized geometries of the reaction pathways. The MM atoms were considered as point charges only, and therefore the additional imaginary frequencies corresponding to the approximate Hessian were excluded. ${ }^{61}$ These frequencies were consistently the same both for the RS and TS geometries, whereas additional imaginary frequencies with the larger intensity peaks were present in the TS structures only. The vibrational motion of these TS frequencies corresponded to the bond breaking and forming coordinates. We estimated the solvent kinetic isotope effects (solvent KIEs) by performing calculations using standard proton mass as well as using deuterated water molecules. Based on the estimated free energy difference $\left(\Delta \Delta G^{\ddagger}\right)$ between the calculated zero-point vibrational energies for the $\mathrm{D}_{2} \mathrm{O} / \mathrm{H}_{2} \mathrm{O}$ systems at the TS/RS, we obtained the solvent kinetic isotope effects of about 1.8 to 3.2 for the WT (corresponding to the TSa: $\mathrm{Q}=0.4 \AA$ and TSb: $\mathrm{Q}=0.6$ $\AA$ structures, respectively), 1.4 to 2.2 for the H145A (corresponding to the optimized structures within the range of $\mathrm{Q}=0.2$ to $0.8 \AA$ ), and $1.01-1.02$ for the T138Stop systems (corresponding to TSb: $\mathrm{Q}=0.0 \AA$ and TSa: $\mathrm{Q}=-0.2 \AA$ structures, respectively). The results suggest the loss of solvent KIEs for the nearly inactive T138Stop mutant.

Downhill trajectories. As an additional test of the mechanistic reaction pathway found with $\mathrm{QM} / \mathrm{MM}$ minimizations, we ran sets of downhill QM/MM trajectories. Geometry optimizations in proteins are well known to suffer from being trapped in some of the myriads of local minima, and free energy calculations are necessary for more quantitative results. ${ }^{62-63}$ Here we tested whether our minimized pathways correspond to the ones obtained by dynamical trajectories. We initiated random downhill trajectories from near the transition states for the WT enzyme and the T138Stop mutant, starting from different thermally equilibrated classical regions. The obtained trajectories initiated at conformations close to the transition state (Figure 6) indeed terminated both at the reactant side and the product side, confirming that the starting structures were part of the TS ensemble.

For the WT system, a total of 86 simulations were initiated from the QM/MM optimized structures at the $Q=0.4 \AA$ (66 trajectories, all $100 \mathrm{fs}$ long), and $Q=0.6 \AA$ (20 trajectories of length between 317 and $584 \mathrm{fs}$ ) values of the reaction coordinate (Figure 5, TSa and TSb, respectively). Out of the 66 trajectories initiated at $Q=0.4 \AA$ (closer to the reactants, TSa), 4 remained near the transition state, 14 reached the product state, and 48 the reactant state. Out of the 20 trajectories, initiated at $Q=0.6 \AA$ (closer to product, TSb), 12 reached the product state, and 8 the reactant state. 
For the T138Stop system, 20 trajectories were initiated both at $Q=-0.2 \AA$ (Figure 5, TSa), and also at $Q=0.0 \AA$, at the free energy barrier maximum (Figure 5, TSb). Trajectories were run between 70 and 1000 fs long, each dynamics reaching reaction coordinate values either below $Q=-1.47 \AA$ (RS) or above $Q=1.61 \AA$ (PS). 14 out of the 20 trajectories reached the product state, and 6 the reactant state starting from $Q=0.0 \AA$, and 12 reached the reactant state, 8 the product state starting at $Q=-0.2 \AA$. Note that we have carried out both forward and backward minimizations using our complex reaction coordinate to avoid hysteresis as much as possible. $^{42}$

Note also that the downhill reaction pathways for WT and T138Stop are initiated near the maxima of the corresponding energy profiles. These positions, do not necessarily have to coincide with the actual TS locations (i.e., having a splitting probability of 0.5 ) along the reaction coordinate $\mathrm{Q},{ }^{64}$ though in this case they are clearly very close.

We obtained a very good correlation between the reaction pathway calculated by QM/MM minimizations and the ensemble of pathways obtained via downhill trajectories (Figure 5). We could also confirm the distinct difference between the reaction pathways for the WT and T138Stop enzymes, with the latter being much more stepwise.

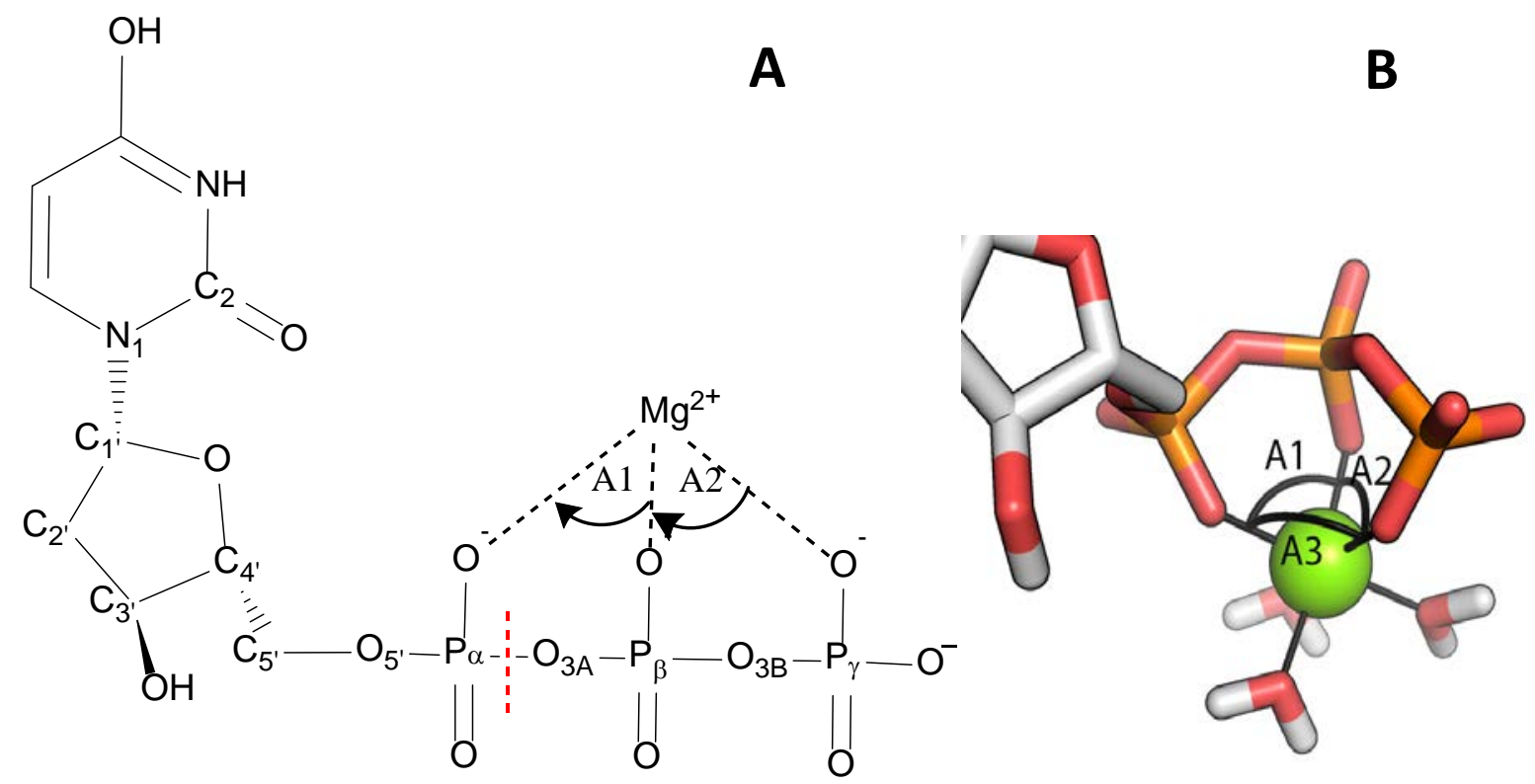

Figure 8. A) Schematic view of the dUTP molecule coordinated to the $\mathrm{Mg}^{2+}$ ion. B) The hexacoordinated $\mathrm{Mg}^{2+}$ ion, as bound in the active site. Definition of the three angles, $\mathrm{A1}$, A2, and A3, are also shown.

$\mathbf{M g}^{2+}$-ion coordination. $\mathrm{Mg}^{2+}$ is an essential cofactor in most phosphoryl transfer and hydrolysis catalyzing enzymes. Its role in modulating the catalytic reaction, however, is not yet well understood. We investigated the $\mathrm{Mg}^{2+}$ coordination, in particular, focusing on the OMg-O angles during the reaction (Figures 8 and S5). Three angles, labeled A1, A2 and A3 (Figure 8B) are most relevant to characterizing the phosphate - magnesium binding geometry: (i) A1, between the $\alpha$ - and $\beta$-phosphates and the $\mathrm{Mg}^{2+}$ (O1A-Mg-O1B), and (ii) A2, between the $\beta$ - and $\gamma$-phosphates and the $\mathrm{Mg}^{2+}$ (O1B-Mg-O2G), and (iii) A3, between the $\alpha$ - and $\gamma$ phosphates and the $\mathrm{Mg}^{2+}(\mathrm{O} 1 \mathrm{~A}-\mathrm{Mg}-\mathrm{O} 2 \mathrm{G})$. The increase in A1 and A2 (Figure S5) correlates strongly with the changes in the reaction coordinate, whereas A3 changes only in case of the 
T138Stop mutant. A1 and A2 reach the ideal $90^{\circ}$ angle - required for the symmetric octahedral $\mathrm{Mg}^{2+}$ coordination - right before the transition state region of the reaction pathway. A3, on the other hand, remains at around $100^{\circ}$ throughout the reaction profile for the WT and H145A enzymes (Figure S5), thus causing a small distortion from an ideal octahedral Mg-coordination. The $\gamma$-phosphate in the T138Stop mutant is not coordinated by key protein residues observed in the WT, due to the lack of the C-terminal arm. Accordingly, as compared with the wild type, the $\mathrm{Mg}^{2+}$ coordination in this mutant leads to the largest differences in the values of A3 (Figure S5), an angle involving the $\gamma$-phosphate.

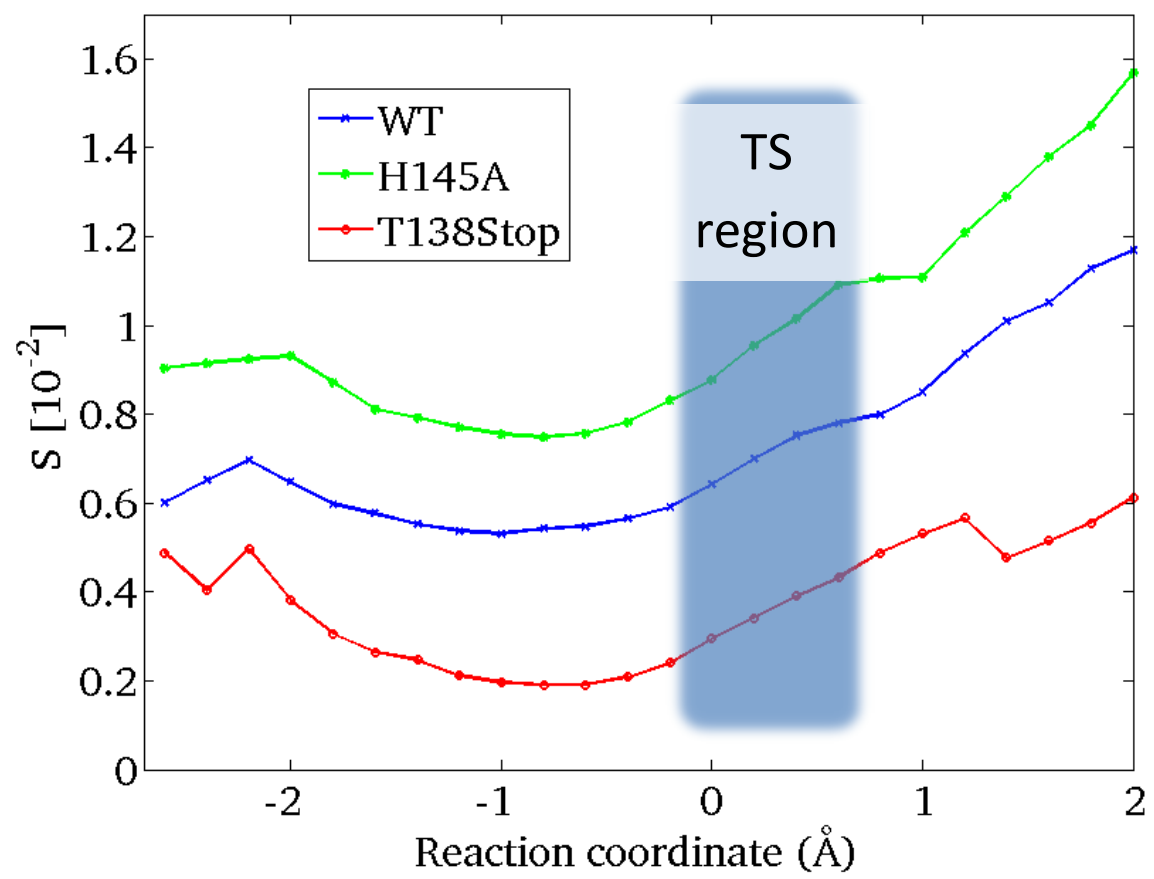

Figure 9. Symmetry parameter (S) along the reaction profile. $\mathrm{Mg}^{2+}$ coordination becomes most symmetric (i.e., smaller $S$ values) before the transition state region (TS, blue area) is reached. A more symmetrical $\mathrm{Mg}^{2+}$-coordination could drive the reaction towards the TS region. $S$ is defined according to Eq. (1).

Continuous symmetry measure. To provide a generic, quantitative analysis tool measuring the changes in the $\mathrm{Mg}^{2+}$ coordination (i.e., the symmetry) along the reaction profile, we implemented a simple algorithm to calculate a continuous symmetry measure ${ }^{34}$ around the $\mathrm{Mg}^{2+}$. We use this symmetry measure S (see Eq. 1) to follow the changes along catalytic reactions. Previously, symmetry measures have been applied to a variety of systems, such as studies of interconversion paths in transition metal chemistry. ${ }^{65-67}$ We determined the overall coordination symmetry in the three different systems using the continuous symmetry measure S (Figure 9). Interestingly, all systems display the same general trend: the coordination becomes gradually more symmetrical as the reaction progresses, and a minimum in $\mathrm{S}$ is reached before the transition state. After this point, the symmetry measure $S$ increases again, as the product state is reached. Interestingly, the T138Stop mutant has the most relaxed and symmetrical $\mathrm{Mg}^{2+}$ coordination compared with the WT and H145A, due to the lack of the Cterminal arm, which would coordinate the phosphate chain of dUTP (Figure 9). Reaching more symmetrical coordination around the $\mathrm{Mg}^{2+}$ should energetically stabilize the TS compared with the reactant state (RS), and the lack of this difference in the stabilization between RS and TS may lead to decreased activity. In general, however, it is hard to quantify the energetic contribution arising due only to the changes in the $\mathrm{Mg}^{2+}$-coordination. Based on 
thorough gas-phase and solution studies by the Florián group ${ }^{68}$ analyzing the energetics of distortions in metal ion coordination, the energetic stabilization may be estimated on the order of $2 \mathrm{kcal} / \mathrm{mol}$. To sum up, the $\mathrm{Mg}^{2+}$-coordination symmetry varies along the reaction coordinate consistently in all our three systems, and thus the metal coordination symmetry emerges as a possible contributing factor to the catalytic mechanism.

Role of the C-terminal arm in substrate specificity. Interestingly, the particularly important role of the A2 and A3 angles involving the $\gamma$-phosphate (Figure 8B) could contribute to the dUDP/dUTP specificity via the C-terminal arm, preventing the dUDP hydrolysis while allowing it for dUTP. For E. coli enzymes, it was found that the flexible C-terminal arm takes up an ordered structure only in the presence of the $\gamma$-phosphate, ${ }^{29-30}$ whereas other organisms such as the human dUTPase may have a C-terminal arm located in the vicinity of the active site, even when a bound substrate is absent. ${ }^{69}$ A recent mutational study demonstrated that the C-terminal arm P-loop-like motif is required for catalytic activity and it also helps to discriminate against dUDP substrates. ${ }^{17}$ The differences we observed in angles A2 and A3 along the catalytic reaction (Figure S5) with the C-terminal arm present (WT and H145A) and absent (T138Stop) are also consistent with distinct changes in the dihedral angles of the triphosphate chain. Three relevant dihedral angles of the triphosphate backbone, $\mathrm{O}_{5},-\mathrm{P}_{\alpha}-\mathrm{O}_{3 A^{-}}$ $\mathrm{P}_{\beta}(\mathrm{A}), \mathrm{P}_{\alpha}-\mathrm{O}_{3 \mathrm{~A}}-\mathrm{P}_{\beta}-\mathrm{O}_{3 \mathrm{~B}}$ (B) and $\mathrm{O}_{3 \mathrm{~A}}-\mathrm{P}_{\beta}-\mathrm{O}_{3 \mathrm{~B}}-\mathrm{P}_{\gamma}$ (C) (Figure S6), show a distinctly different orientation of the chain for the T138Stop mutant as compared to the WT and H145A. The same trend is also observed in the original crystallographic structures with non-hydrolysable ligands (Table S1 and Figure S2A). This may offer new mechanistic insights about the role of the C-terminal arm structural motif in the dUDP/dUTP specificity.

Analogy with two-metal ion catalysis. In many enzymes that carry out phosphoryl transfer and hydrolysis, the reacting phosphate groups are coordinated via divalent metal ions, in the active site, mainly $\mathrm{Mg}^{2+}$ (Figure 10). A common ubiquitous two-metal ion catalytic mechanism was proposed for polymerases and nucleases by Steitz and Steitz. ${ }^{70}$ In this mechanism, metal ion A coordinates the nucleophile, whereas metal ion B coordinates the leaving group. Similar to dUTPases, only a single metal ion is involved in the catalytic reaction of several enzymes, such as nucleases with the $\beta \beta \alpha$-Me structural motifs, ${ }^{71-72}$ or HUH motifs (where H's denote two histidine residues separated by a hydrophobic residue, U). ${ }^{69,}$ 73-74 An analogous role of the single metal ion in one-metal ion catalysis has been proposed to metal ion B in two-metal ion catalysis based on structural data. ${ }^{8}$ An additional emerging role for metal ion $\mathrm{B}$ is to take on a more symmetrical coordination near the transition state region, and thereby to facilitate catalysis. ${ }^{8,} 43$ These roles show great similarities to the roles of metal ion B in two metal ion catalysis. ${ }^{43}$ 


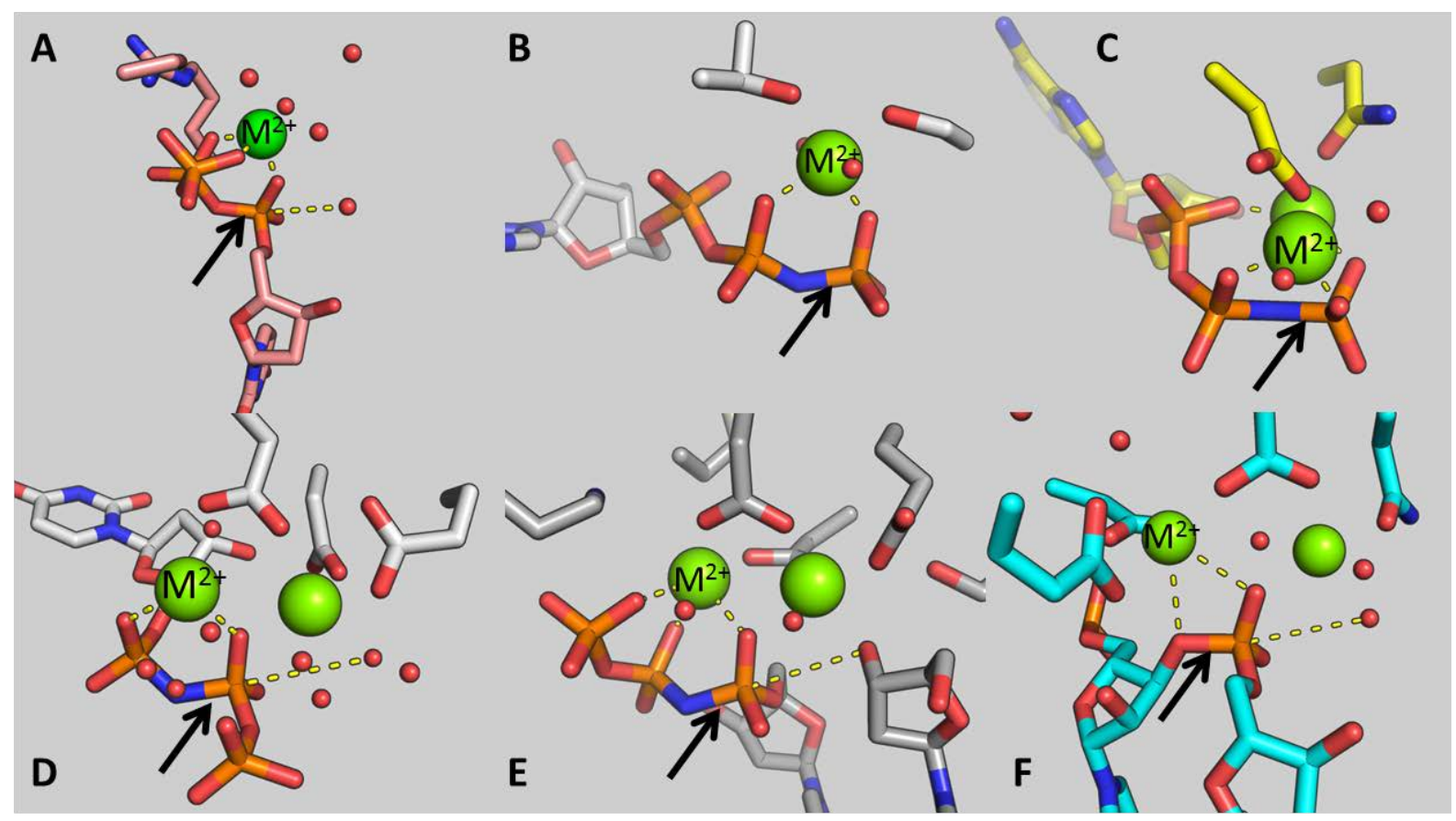

Figure 10. Equivalent active-site metal ion $\left(\mathbf{M}^{2+}\right)$ positions in various phosphate processing enzymes. The residues within $4 \AA$ around the metal ions (green spheres) are shown as sticks. Prototype $\mathrm{Mg}^{2+}$ coordination geometries are presented for trimeric dUTPases (A, PDB ID: 2PY4), ATPases (B, PDB ID: 3ZFD), Ser/Thr kinases (C, PDB ID: 4DFX), dimeric dUTPases (D, PDB ID: 2CIC), polymerases (E, PDB ID: 4Q8E), and RNases H (F, PDB ID: 1ZBL). The scissile $\mathrm{P}-\mathrm{O}$ bonds (or its non-hydrolyzable equivalents) are highlighted by arrows.

An analogous metal ion is also present in most AAA+ ATPases coordinating the ATP at the $\beta$-, and $\gamma$-phosphates, but not the $\alpha$-phosphates (Figure 10B). Related phosphate transfer reactions are carried out by kinases cleaving ATP and transferring the $\gamma$-phosphate to an alcohol group of an amino acid side chain (typically Ser, Thr, or Tyr). In this important family of enzymes, two $\mathrm{Mg}^{2+}$ ions are commonly observed in high resolution crystal structures, with a consensus structure for Ser/Thr kinases where one of the metal ions coordinates $\alpha-, \beta-$, and the $\gamma$-phosphates, whereas the second metal ion coordinates $\beta$-, and $\gamma$-phosphates, as in for example cyclic AMP-dependent protein kinase (PKA, Figure 10C). ${ }^{75}$ Intriguingly, both metal ions coordinate the cleaved $\gamma$-phosphate of the ATP for these catalytic reactions, whereas none coordinate the attacking oxygen nucleophile in these highly specific enzymes, just as in dUTPases. The role of the two metal ions in these kinases therefore resembles the role of metal ion B in two-metal ion catalysis, and the single metal ion observed in one-metal ion catalysis in ATPases and dUTPase. Interestingly, the $\mathrm{Mg}^{2+}$ to $\mathrm{Ca}^{2+}$ substitution effects are typically also similarly inhibitory ${ }^{76}$ for two-metal ion catalysis as well as for the one-metal ion catalysis in dUTPase ${ }^{21-22}$ and in several ATPases ${ }^{77-81}$ and kinases ${ }^{78,82-83}$ with some notable exceptions. $^{84-85}$

This key metal ion often coordinates the cleaved phosphate. This helps the catalysis by lowering the $\mathrm{p} K_{\mathrm{a}}$ of the phosphate group. As the overall effect of this $\mathrm{Mg}^{2+}$ coordination, the $\alpha$-phosphate oxygen atoms are more acidic, and thus their protonation is significantly less favorable. Accordingly, the single most likely proton transfer pathway corresponds to the protonation of the Asp83, and protonation of the $\alpha$-phosphate is unfavorable. 
The prominent role of the $\mathrm{Mg}^{2+}$ ion in lowering of the $\mathrm{p} K_{\mathrm{a}}$ of its ligands is also important for the second ion in two-metal ion catalysis. In this case, the second $\mathrm{Mg}^{2+}$ ion typically coordinates the nucleophilic water (or the attacking group, in general). This was observed to lead to a mechanism with early proton transfer, preceding the coupled phosphate cleavage in the RNase $\mathrm{H}$ catalytic reaction. ${ }^{43,76}$ Likewise, early proton transfer was found to take place in the two metal ion-catalyzed reactions of DNA polymerases ${ }^{86}$ and adenylyl cyclases. ${ }^{87}$ In onemetal ion catalysis, the unligated nucleophilic water has a higher $\mathrm{p} K_{\mathrm{a}}$, which explains the difference in the mechanism leading to the late proton transfer observed in this study as well.

A key consensus role thus emerges for a functional $\mathrm{Mg}^{2+}$ ion that coordinates the cleaved phosphate. The specific active site geometry and fine-tuning of the $\mathrm{p} K_{\mathrm{a}}-\mathrm{s}$ of the catalytic groups by the metal ion leads to a reaction mechanism that is very different from the corresponding slow, uncatalyzed reaction in water. ${ }^{88-89}$ Thus, the $\mathrm{Mg}^{2+}$ ion appears to have an essential contribution for the highly specific catalytic reactions, by (i) lowering the $\mathrm{p} K_{\mathrm{a}}$ of the leaving group, and (ii) imposing a specific constrained geometry for the triphosphate chain, which drives the reaction towards its transition state and products.

Conserved $\pi-\pi$ stacking interaction with the NTP nucleobase. Despite the relatively large ( $\sim 7 \AA$ ) distance of the $\mathrm{H} 145$ residue from the $\mathrm{P}_{\alpha}$ cleavage site, this residue has a significant contribution to the catalytic activity. It establishes a $\pi-\pi$ stacking interaction with the uracil ring (Figure 1B), ${ }^{25}$ taking up a T-shaped orientation of the rings. ${ }^{90}$ This well-conserved interaction is also observed in many other trinucleotide-binding enzymes, including $\mathrm{ABC}$ transporters, ${ }^{91}$ kinesins $^{92}$ or kinases. ${ }^{93}$ The general importance of aromatic residues corresponding to the H145 amino acid across many dUTPases was established in a study of 500 dUTPase sequences. ${ }^{25}$ Amongst these 500 structures, the most conserved residue is Phe (96.4\% of cases), whereas His accounted for $1.8 \%$ of the structures. Based on our measurements and on the kinetic measurements reported, ${ }^{25}$ the catalytic rate was affected when the aromatic residue was mutated to an Ala, whereas the substrate binding remained relatively intact as compared to the wild type. The loss of catalytic activity was about 10 to 20 fold, ${ }^{25}$ both in the human and in the $M$. tuberculosis enzymes. There is thus an outstanding puzzling question as to how the conserved $\pi-\pi$ stacking interaction affects the catalytic reaction, from such a remote distance to the cleavage site.

Charge distribution of the QM regions at the TS. T-shaped $\pi-\pi$ stacking interactions are thought to have more electrostatic character compared with sandwich-shaped orientations. ${ }^{94}$ To better understand whether the $\pi-\pi$ stacking interactions with the nucleobase may produce additional charge transfer that in turn would promote catalysis, we calculated the partial charges for the minimized structures. We compared the charge distribution in the WT with the mutants, in their respective TS conformations. It is important to emphasize that the charge distribution of the high energy TS is the main determining factor of the catalytic barrier, whereas the charge distribution at the RS is less relevant for predicting reactivity. For both mutants, we calculated the difference in the distribution of partial charges, as illustrated in Figure 11 using a color coding where blue means that the group is more negative than the WT, red means that the group is more positive, and white means no change in overall charge. The color code ranges between -0.2 and $0.2 e$. The numerical values are also given in Table S3.

We observed that the uracil ring has a significantly altered polarization due to the missing $\pi-\pi$ stacking interaction, and this also affects the sugar moiety (Figure 11 and Table S3). However, the geometric differences corresponding to the H145A mutation are likely the main reason for the decreased activity of the H145A mutant (SI text). 
Intriguingly, both mutants have similar changes in the polarization of the active site at the TS geometries. We find that the largest change is observed in the polarization of the relatively distant $\gamma$-phosphate, interestingly, which becomes more negative at the H145A and T138Stop enzymes compared to the WT TS. Likewise, the uracil group also becomes more negative at the TS in the H145A and T138Stop mutants compared with the WT, whereas the deoxyribose and the $\alpha$-phosphate groups have a more positive partial charge in the mutant enzymes.

A

H145A
B

T138Stop
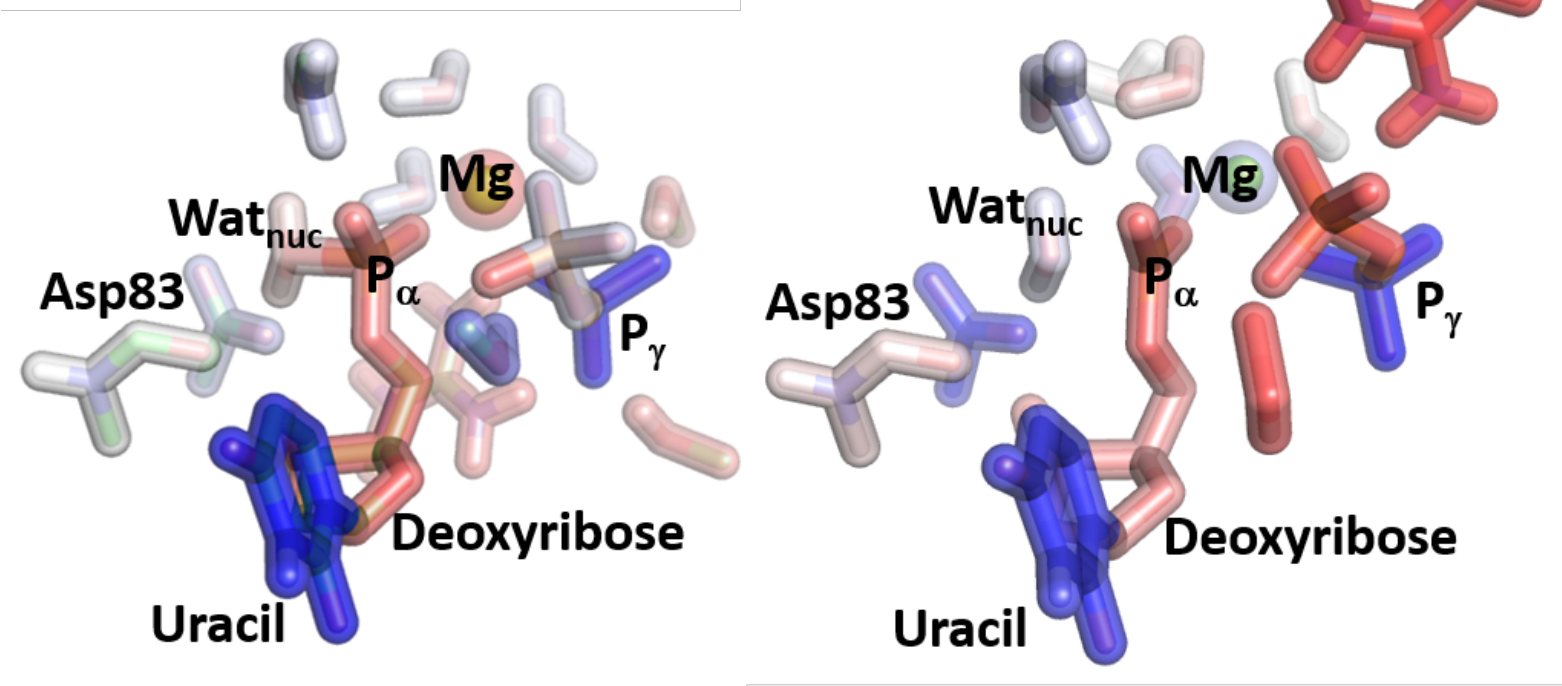

Figure 11. Charge distribution difference for the QM region of (A) H145A and, (B) T138Stop mutants compared with the WT enzyme, using the CHELPG atomic charge scheme as implemented in Gaussian09. The atoms are displayed in solid sticks using a color scheme based on the elements, and with wider transparent sticks using a bluewhite-red color scheme, in the range of $-0.2 e$ to $+0.2 e$ (scale bar in atomic units), based on the charge difference for the group of atoms between the (A) H145A or (B) T138Stop simulation and the WT. For definition of the atomic groups and the numerical values for the charge differences, see Table S3.

\section{Conclusions}

Phosphate cleavage and transfer reactions are universal in all biological processes of all living organisms. Furthermore, phosphate-based reaction mechanisms have many common aspects shared amongst the majority of enzymes that carry out the corresponding catalytic reactions, such as the use of divalent $\mathrm{Mg}^{2+}$ ions. ${ }^{35}$

We investigated here the phosphate cleavage reaction in the WT and two mutant forms (H145A and T138Stop) of dUTPase enzyme complexes. Our analysis of steady-state 
activities of the WT and T138Stop and H145A mutants showed that the activity of the H145A mutant is lower by one order of magnitude, while that of the T138Stop mutant is lower by three orders of magnitude, as compared to the WT, in agreement with the homologous mutation in the human dUTPase enzyme. ${ }^{17}$ To understand what the key factors are that might control the observed changes in the catalytic activities, we studied the reaction mechanism of proton transfer and phosphate cleavage using QM/MM methodology. We found a one-step associative $A_{N} D_{N}$ pathway for the catalytic mechanism with a loose dissociative TS in all three systems. The dUTPase hydrolysis of the $\alpha$-phosphate also involves a coupled proton transfer from the nucleophilic water to the catalytic Asp83 residue. The coupled proton transfer occurred in a single step with the phosphate cleavage in the following sequence of events. First, the nucleophilic water approached the phosphate. Subsequently, the $\mathrm{P}_{\alpha}-\mathrm{O}_{3 \mathrm{~A}}$ bond broke. At the TS in the WT enzyme, the new $\mathrm{P}_{\alpha}-\mathrm{O}_{\text {wat }}$ bond formed at the same time as the proton transfer from the water to the Asp83 was initiated. The final products were then reached by completing the proton transfer. Essentially, we observed the same mechanism taking place in all the three systems. The main difference was that the proton transfer was decoupled from the TS in the two mutants as confirmed by NBO analysis, and it happened particularly late in the nearly inactive T138Stop mutant. We did not observe major differences in the ligand binding, protein stability, or in the structures of the assembled complexes. However, the degree of concertedness of the proton transfer and the phosphate bond formation at the TS were correlated strongly with the known catalytic activities. We observed less concerted proton transfer in case of the H145A mutant, and further significantly decreased concertedness for the T138Stop mutant. Our calculations also suggest the loss of solvent KIEs for the T138Stop mutant. Our calculated reaction barriers are also in agreement with the catalytic rates measured in our corresponding site-directed mutagenesis experiments. The observed concerted coupled proton transfer appears to be a general mechanism in enzymes that catalyzes phosphate transfer and cleavage reactions. ${ }^{43,} 76,95-98$

Our calculations also provided evidence that the specific metal ion coordination mode may play a prominent role in the catalytic reaction. We developed a new implementation to quantitatively determine the symmetry around the metal ion during the catalytic reaction. Based on our analysis using the calculated continuous symmetry measure ${ }^{34}$ of the metal ion coordination throughout the reaction, a symmetric, octahedral $\mathrm{Mg}^{2+}$ coordination was shown to stabilize the TS for all cases. All three systems displayed the same trend for the change in the metal-coordination symmetry along the reaction pathway. The T138Stop mutant had the most symmetrical, thus most relaxed coordination around the $\mathrm{Mg}^{2+}$ throughout the reaction, due to the lack of the C-terminal arm. Changes in the charge distributions of the three systems in their respective TS, also revealed significant differences in the partial charge transfer from the protein to the substrate, due to the missing native protein-protein and protein-substrate interactions in the mutants. The uracil group has a larger partial negative charge in the mutant structures at the TS compared to the WT, whereas deoxyribose is more positively polarized. Interestingly, the negative polarization of the $\gamma$-phosphate is the largest change between the partial charges of the TS structures. Geometrical relaxation of the active site due to mutations could play a key role in altering the TS-s, leading to a more decoupled proton transfer. The key emerging roles for a functional $\mathrm{Mg}^{2+}$ ion that coordinates the phosphate leaving group are (i) to lower the $\mathrm{p} K_{\mathrm{a}}$ of the cleaved phosphate groups, and (ii) to impose constrained coordination geometry on the $\mathrm{Mg}^{2+}$, which drives the reaction towards the transition state and product.

The structural and electronic changes involved in the dUTPase catalytic reaction evidenced here, in conjunction with the experimental data, shed new light on the crucial role of the 
dUTPase C-terminal arm in modulating ligand binding geometry and catalytic activity and of the essential roles of the $\mathrm{Mg}^{2+}$ ion cofactor. Detailed understanding of the reaction process provided by this study could lead to the design of new drugs, and new approaches to rationally control the activity of dUTPase and other (e.g., ATPase, kinase or polymerase) enzymes.

\section{Acknowledgments}

This work was supported by the Intramural Research Program of the National Institutes of Health, the Hungarian Scientific Research Fund (OTKA NK 84008, K109486), the Baross program of the New Hungary Development Plan (3DSTRUCT, OMFB-00266/2010 REGKM-09-1-2009-0050), the Hungarian Academy of Sciences (TTK IF-28/2012), and the European Commission FP7 Biostruct-X project (contract number 283570). This work used the computational resources of the NIH biowulf cluster, and the CCPBioSim via the ARCHER UK National Supercomputing Service. AL was awarded the student travel grant of the Budapest University of Technology and Economics. PKS thanks Prof. Sun Choi at Ewha Womans University for laboratory facilities, and the Korean Federation of Science and Technology Societies (KOFST) for a research fellowship under the Brain Pool program.

\section{Supporting Information Available}

Supporting information consists of six additional figures (Figures S1-S6), three tables of data (Tables S1-S3) and two paragraphs on the protonation state of H145 and on the structural and polarization effect of the H145A mutation. This information is available free of charge via the Internet at http://pubs.acs.org/. 


\section{References}

(1) McIntosh, E. M.; Ager, D. D.; Gadsden, M. H.; Haynes, R. H. Proceedings of the National Academy of Sciences 1992, 89, 8020-8024.

(2) Björnberg, O.; Neuhard, J.; Nyman, P. O. Journal of Biological Chemistry 2003, 278, 20667-20672.

(3) Johansson, E.; Björnberg, O.; Nyman, P. O.; Larsen, S. Journal of Biological Chemistry 2003, 278, 27916-27922.

(4) Huffman, J. L.; Li, H.; White, R. H.; Tainer, J. A. Journal of Molecular Biology 2003, 331, 885-896.

(5) Li, H.; Xu, H.; Graham, D. E.; White, R. H. Journal of Biological Chemistry 2003, 278, 11100-11106.

(6) Helt, S. S.; Thymark, M.; Harris, P.; Aagaard, C.; Dietrich, J.; Larsen, S.; Willemoes, M. Journal of Molecular Biology 2008, 376, 554-569.

(7) Vertessy, B. G.; Toth, J. Acc Chem Res 2009, 42, 97-106.

(8) Yang, W. Nat. Struct. Mol. Biol. 2008, 15, 1228-1231.

(9) Mustafi, D.; Bekesi, A.; Vertessy, B. G.; Makinen, M. W. Proc Natl Acad Sci U S A 2003, 100, 56705.

(10) Chan, S.; Segelke, B.; Lekin, T.; Krupka, H.; Cho, U. S.; Kim, M.-y.; So, M.; Kim, C.-Y.; Naranjo, C. M.; Rogers, Y. C.; Park, M. S.; Waldo, G. S.; Pashkov, I.; Cascio, D.; Perry, J. L.; Sawaya, M. R. Journal of Molecular Biology 2004, 341, 503-517.

(11) Tarbouriech, N.; Buisson, M.; Seigneurin, J.-M.; Cusack, S.; Burmeister, W. P. Structure 2005, 13, 1299-1310.

(12) Camacho, A.; Arrebola, R.; Pena-Diaz, J.; Ruiz-Perez, L. M.; Gonzalez-Pacanowska, D. Biochem J 1997, 325 ( Pt 2), 441-7.

(13) Moroz, O. V.; Harkiolaki, M.; Galperin, M. Y.; Vagin, A. A.; Gonzalez-Pacanowska, D.; Wilson, K. S. J Mol Biol 2004, 342, 1583-97.

(14) Hemsworth, G. R.; Moroz, O. V.; Fogg, M. J.; Scott, B.; Bosch-Navarrete, C.; GonzalezPacanowska, D.; Wilson, K. S. J Biol Chem 2011, 286, 16470-81.

(15) Takacs, E.; Nagy, G.; Leveles, I.; Harmat, V.; Lopata, A.; Toth, J.; Vertessy, B. G. FEBS Lett 2010, 584, 3047-54.

(16) Fiser, A.; Vertessy, B. G. Biochem Biophys Res Commun 2000, 279, 534-42.

(17) Pécsi, I.; Szabó, J. E.; Adams, S. D.; Simon, I.; Sellers, J. R.; Vértessy, B. G.; Tóth, J. Proc. Natl. Acad. Sci. USA 2011, 108, 14437-14442.

(18) Walker, J. E.; Saraste, M.; Runswick, M. J.; Gay, N. J. The EMBO journal 1982, 1, 945-951.

(19) Ogura, T.; Wilkinson, A. J. Genes to Cells 2001, 6, 575-597.

(20) Hanson, P. I.; Whiteheart, S. W. Nat Rev Mol Cell Biol 2005, 6, 519-529.

(21) Hidalgo-Zarco, F.; Camacho, A. G.; Bernier-Villamor, V.; Nord, J.; Ruiz-Perez, L. M.; GonzalezPacanowska, D. Protein Sci 2001, 10, 1426-33.

(22) Persson, R.; McGeehan, J.; Wilson, K. S. Protein Expr Purif 2005, 42, 92-9.

(23) Harris, J. M.; McIntosh, E. M.; Muscat, G. E. O. Journal of Molecular Biology 1999, 288, 275-287.

(24) Warshel, A.; Levitt, M. J. Mol. Biol. 1976, 103, 227-249.

(25) Pecsi, I.; Leveles, I.; Harmat, V.; Vertessy, B. G.; Toth, J. Nucleic Acids Res 2010, 38, 7179-86.

(26) Varga, B.; Barabas, O.; Takacs, E.; Nagy, N.; Nagy, P.; Vertessy, B. G. Biochem Biophys Res Commun 2008, 373, 8-13.

(27) Mol, C. D.; Harris, J. M.; McIntosh, E. M.; Tainer, J. A. Structure 1996, 4, 1077-92.

(28) Shao, H.; Robek, M. D.; Threadgill, D. S.; Mankowski, L. S.; Cameron, C. E.; Fuller, F. J.; Payne, S. L. Biochim Biophys Acta 1997, 1339, 181-91.

(29) Vertessy, B. G. Proteins 1997, 28, 568-79.

(30) Vertessy, B. G.; Larsson, G.; Persson, T.; Bergman, A. C.; Persson, R.; Nyman, P. O. FEBS Lett 1998, 421, 83-8.

(31) Nord, J.; Kiefer, M.; Adolph, H. W.; Zeppezauer, M. M.; Nyman, P. O. FEBS Lett 2000, 472, 312-6. 
(32) Freeman, L.; Buisson, M.; Tarbouriech, N.; Van der Heyden, A.; Labbe, P.; Burmeister, W. P. J Biol Chem 2009, 284, 25280-9.

(33) Barabás, O.; Németh, V.; Bodor, A.; Perczel, A.; Rosta, E.; Kele, Z.; Zagyva, I.; Szabadka, Z.; Grolmusz, V. I.; Wilmanns, M.; Vértessy, B. G. Nucleic Acids Research 2013, 41, 10542-10555.

(34) Zabrodsky, H.; Peleg, S.; Avnir, D. Journal of the American Chemical Society 1992, 114, 78437851.

(35) Palermo, G.; Cavalli, A.; Klein, M. L.; Alfonso-Prieto, M.; Dal Peraro, M.; De Vivo, M. Acc Chem Res 2015.

(36) Prasad, G. S.; Stura, E. A.; Elder, J. H.; Stout, C. D. Acta Crystallogr D Biol Crystallogr 2000, 56, 1100-9.

(37) Brooks, B. R.; Bruccoleri, R. E.; Olafson, B. D.; States, D. J.; Swaminathan, S.; Karplus, M. J. Comput. Chem. 1983, 4, 187-217.

(38) Jo, S.; Kim, T.; Iyer, V. G.; Im, W. Journal of Computational Chemistry 2008, 29, 1859-1865.

(39) Shao, Y.; Molnar, L. F.; Jung, Y.; Kussmann, J.; Ochsenfeld, C.; Brown, S. T.; Gilbert, A. T.; Slipchenko, L. V.; Levchenko, S. V.; O'Neill, D. P.; DiStasio, R. A., Jr.; Lochan, R. C.; Wang, T.; Beran, G. J.; Besley, N. A.; Herbert, J. M.; Lin, C. Y.; Van Voorhis, T.; Chien, S. H.; Sodt, A.; Steele, R. P.; Rassolov, V. A.; Maslen, P. E.; Korambath, P. P.; Adamson, R. D.; Austin, B.; Baker, J.; Byrd, E. F.; Dachsel, H.; Doerksen, R. J.; Dreuw, A.; Dunietz, B. D.; Dutoi, A. D.; Furlani, T. R.; Gwaltney, S. R.; Heyden, A.; Hirata, S.; Hsu, C. P.; Kedziora, G.; Khalliulin, R. Z.; Klunzinger, P.; Lee, A. M.; Lee, M. S.; Liang, W.; Lotan, I.; Nair, N.; Peters, B.; Proynov, E. I.; Pieniazek, P. A.; Rhee, Y. M.; Ritchie, J.; Rosta, E.; Sherrill, C. D.; Simmonett, A. C.; Subotnik, J. E.; Woodcock, H. L., 3rd; Zhang, W.; Bell, A. T.; Chakraborty, A. K.; Chipman, D. M.; Keil, F. J.; Warshel, A.; Hehre, W. J.; Schaefer, H. F., 3rd; Kong, J.; Krylov, A. I.; Gill, P. M.; Head-Gordon, M. Phys Chem Chem Phys 2006, 8, 3172-91.

(40) Becke, A. The Journal of Chemical Physics 1993, 98, 5648-5652.

(41) Woodcock, H. L., 3rd; Hodoscek, M.; Gilbert, A. T.; Gill, P. M.; Schaefer, H. F., 3rd; Brooks, B. R. J Comput Chem 2007, 28, 1485-502.

(42) Rosta, E.; Woodcock, H. L.; Brooks, B. R.; Hummer, G. J Comput Chem 2009, 30, 1634-41.

(43) Rosta, E.; Nowotny, M.; Yang, W.; Hummer, G. J Am Chem Soc 2011, 133, 8934-41.

(44) Chu, J.-W.; Trout, B. L.; Brooks, B. R. The Journal of Chemical Physics 2003, 119, 12708-12717.

(45) Frisch, M. J.; Trucks, G. W.; Schlegel, H. B.; Scuseria, G. E.; Robb, M. A.; Cheeseman, J. R.; Scalmani, G.; Barone, V.; Mennucci, B.; Petersson, G. A.; Nakatsuji, H.; Caricato, M.; Li, X.; Hratchian, H. P.; Izmaylov, A. F.; Bloino, J.; Zheng, G.; Sonnenberg, J. L.; Hada, M.; Ehara, M.; Toyota, K.; Fukuda, R.; Hasegawa, J.; Ishida, M.; Nakajima, T.; Honda, Y.; Kitao, O.; Nakai, H.; Vreven, T.; Montgomery, J. A.; Peralta, J. E.; Ogliaro, F.; Bearpark, M.; Heyd, J. J.; Brothers, E.; Kudin, K. N.; Staroverov, V. N.; Kobayashi, R.; Normand, J.; Raghavachari, K.; Rendell, A.; Burant, J. C.; Iyengar, S. S.; Tomasi, J.; Cossi, M.; Rega, N.; Millam, J. M.; Klene, M.; Knox, J. E.; Cross, J. B.; Bakken, V.; Adamo, C.; Jaramillo, J.; Gomperts, R.; Stratmann, R. E.; Yazyev, O.; Austin, A. J.; Cammi, R.; Pomelli, C.; Ochterski, J. W.; Martin, R. L.; Morokuma, K.; Zakrzewski, V. G.; Voth, G. A.; Salvador, P.; Dannenberg, J. J.; Dapprich, S.; Daniels, A. D.; Farkas; Foresman, J. B.; Ortiz, J. V.; Cioslowski, J.; Fox, D. J., Gaussian 09, Revision B.01. Wallingford CT, 2009.

(46) Breneman, C. M.; Wiberg, K. B. Journal of Computational Chemistry 1990, 11, 361-373.

(47) Singh, U. C.; Kollman, P. A. Journal of Computational Chemistry 1984, 5, 129-145.

(48) Pastor, R. W.; Brooks, B. R.; Szabo, A. Molecular Physics 1988, 65, 1409-1419.

(49) Palmen, L. G.; Becker, K.; Bulow, L.; Kvassman, J. O. Biochemistry 2008, 47, 7863-74.

(50) Garcia-Nafria, J.; Timm, J.; Harrison, C.; Turkenburg, J. P.; Wilson, K. S. Acta Crystallographica Section D 2013, 69, 1367-1380.

(51) Barabas, O.; Pongracz, V.; Kovari, J.; Wilmanns, M.; Vertessy, B. G. J Biol Chem 2004, 279, 4290715.

(52) Siggaard, J. H.; Johansson, E.; Vognsen, T.; Helt, S. S.; Harris, P.; Larsen, S.; Willemoes, M. Arch Biochem Biophys 2009, 490, 42-9.

(53) Florián, J.; Warshel, A. The Journal of Physical Chemistry B 1998, 102, 719-734.

(54) Zhang, Z.; Eloge, J.; Florián, J. Biochemistry 2014, 53, 4180-4191. 
(55) Klahn, M.; Rosta, E.; Warshel, A. J. Am. Chem. Soc. 2006, 128, 15310-15323.

(56) Larsson, G.; Nyman, P. O.; Kvassman, J. O. J Biol Chem 1996, 271, 24010-6.

(57) Lassila, J. K.; Zalatan, J. G.; Herschlag, D. Annu Rev Biochem 2011, 80, 669-702.

(58) Di Sabato, G.; Jencks, W. P. J. Am. Chem. Soc. 1961, 83, 4400-4405.

(59) Kirby, A. J.; Varvoglis, A. G. J. Am. Chem. Soc. 1967, 89, 415-423.

(60) Gorenstein, D. G.; Lee, Y. G.; Kar, D. J. Am. Chem. Soc. 1977, 99, 2264-2267.

(61) Ghysels, A.; Woodcock, H. L.; Larkin, J. D.; Miller, B. T.; Shao, Y.; Kong, J.; Neck, D. V.; Speybroeck, V. V.; Waroquier, M.; Brooks, B. R. Journal of Chemical Theory and Computation 2011, 7, 496-514.

(62) Klahn, M.; Braun-Sand, S.; Rosta, E.; Warshel, A. J. Phys. Chem. B 2005, 109, 15645-15650.

(63) Oláh, J.; Mulholland, A. J.; Harvey, J. N. Proceedings of the National Academy of Sciences 2011, $108,6050-6055$.

(64) Berezhkovskii, A. M.; Tofoleanu, F.; Buchete, N.-V. Journal of Chemical Theory and Computation 2011, 7, 2370-2375.

(65) Casanova, D.; Cirera, J.; Llunell, M.; Alemany, P.; Avnir, D.; Alvarez, S. Journal of the American Chemical Society 2004, 126, 1755-1763.

(66) Alvarez, S.; Alemany, P.; Casanova, D.; Cirera, J.; Llunell, M.; Avnir, D. Coordination Chemistry Reviews 2005, 249, 1693-1708.

(67) Tuvi-Arad, I.; Rozgonyi, T.; Stirling, A. The Journal of Physical Chemistry A 2013, 117, 1272612733.

(68) Pontikis, G.; Borden, J.; Martínek, V. c.; Florián, J. The Journal of Physical Chemistry A 2009, 113, 3588-3593.

(69) Tóth, J.; Varga, B.; Kovács, M.; Málnási-Csizmadia, A.; Vértessy, B. G. J. Biol. Chem. 2007 282, $33572-33582$.

(70) Steitz, T. A.; Steitz, J. A. Proc. Natl. Acad. Sci. 1993, 90, 6498.

(71) Friedhoff, P.; Franke, I.; Meiss, G.; Wende, W.; Krause, K. L.; Pingoud, A. Nat Struct Mol Biol 1999, 6, 112-113.

(72) KühImann, U. C.; Moore, G. R.; James, R.; Kleanthous, C.; Hemmings, A. M. FEBS Letters 1999, $463,1-2$.

(73) Ton-Hoang, B.; Guynet, C.; Ronning, D. R.; Cointin-Marty, B.; Dyda, F.; Chandler, M. The EMBO Journal 2005, 24, 3325-3338.

(74) Barabas, O.; Ronning, D. R.; Guynet, C.; Hickman, A. B.; Ton-Hoang, B.; Chandler, M.; Dyda, F. Cell 2008, 132, 208-220.

(75) Bastidas, A. C.; Deal, M. S.; Steichen, J. M.; Keshwani, M. M.; Guo, Y.; Taylor, S. S. Journal of Molecular Biology 2012, 422, 215-229.

(76) Rosta, E.; Yang, W.; Hummer, G. Journal of the American Chemical Society 2014, 136, 31373144.

(77) Beauge, L.; Campos, M. A. Biochim. Biophys. Acta. - Biomembranes 1983, 729, 137-149.

(78) Skou, J. C. Biochim. Biophys. Acta. 1957, 23, 394-401.

(79) Fukushima, Y.; Post, R. L. J. Biol. Chem. 1978, 253, 6853-62.

(80) Knauf, P. A.; Proverbio, F.; Hoffman, J. F. J. Gen. Physiol. 1974, 63, 324-336.

(81) Collins, K.; Sellers, J. R.; Matsudaira, P. J. Cell Biol. 1990, 110, 1137-47.

(82) Ryves, W. J.; Dajani, R.; Pearl, L.; Harwood, A. J. Biochem. Biophys. Res. Commun. 2002, 290, 967-972.

(83) Lu, S.-Y.; Huang, Z.-M.; Huang, W.-K.; Liu, X.-Y.; Chen, Y.-Y.; Shi, T.; Zhang, J. Proteins: Struct., Funct., Bioinf. 2013, 81, 740-753.

(84) Deweese, J. E.; Osheroff, N. Metallomics 2010, 2, 450-459.

(85) Gerlits, O.; Waltman, M. J.; Taylor, S.; Langan, P.; Kovalevsky, A. Biochemistry 2013, 52, 37213727.

(86) Florian, J.; Goodman, M. F.; Warshel, A. J Am Chem Soc 2003, 125, 8163-77.

(87) Mones, L.; Tang, W. J.; Florian, J. Biochemistry 2013, 52, 2672-82.

(88) Duarte, F.; Aqvist, J.; Williams, N. H.; Kamerlin, S. C. J Am Chem Soc 2015, 137, 1081-93. 
(89) Guthrie, J. P. J. Am. Chem. Soc. 1977, 99, 3991-4001.

(90) Meyer, E. A.; Castellano, R. K.; Diederich, F. Angewandte Chemie International Edition 2003, 42, 1210-1250.

(91) Guo, X.; Chen, X.; Weber, I. T.; Harrison, R. W.; Tai, P. C. Biochemistry 2006, 45, 14473-80.

(92) Rickert, K. W.; Schaber, M.; Torrent, M.; Neilson, L. A.; Tasber, E. S.; Garbaccio, R.; Coleman, P. J.; Harvey, D.; Zhang, Y.; Yang, Y.; Marshall, G.; Lee, L.; Walsh, E. S.; Hamilton, K.; Buser, C. A. Arch Biochem Biophys 2008, 469, 220-31.

(93) Boehr, D. D.; Farley, A. R.; Wright, G. D.; Cox, J. R. Chem Biol 2002, 9, 1209-17.

(94) Sinnokrot, M. O.; Sherrill, C. D. J Am Chem Soc 2004, 126, 7690-7.

(95) Lans, I.; Medina, M.; Rosta, E.; Hummer, G.; Garcia-Viloca, M.; Lluch, J. M.; González-Lafont, À. J. Am. Chem. Soc. 2012, 134, 20544-20553.

(96) Castro, C.; Smidansky, E.; Maksimchuk, K. R.; Arnold, J. J.; Korneeva, V. S.; Gotte, M.; Konigsberg, W.; Cameron, C. E. Proceedings of the National Academy of Sciences 2007, 104, 4267-4272.

(97) Ganguly, A.; Thaplyal, P.; Rosta, E.; Bevilacqua, P. C.; Hammes-Schiffer, S. Journal of the American Chemical Society 2014, 136, 1483-1496.

(98) Wang, J.; Yang, W. The Journal of Physical Chemistry B 2013, 117, 9354-9361.

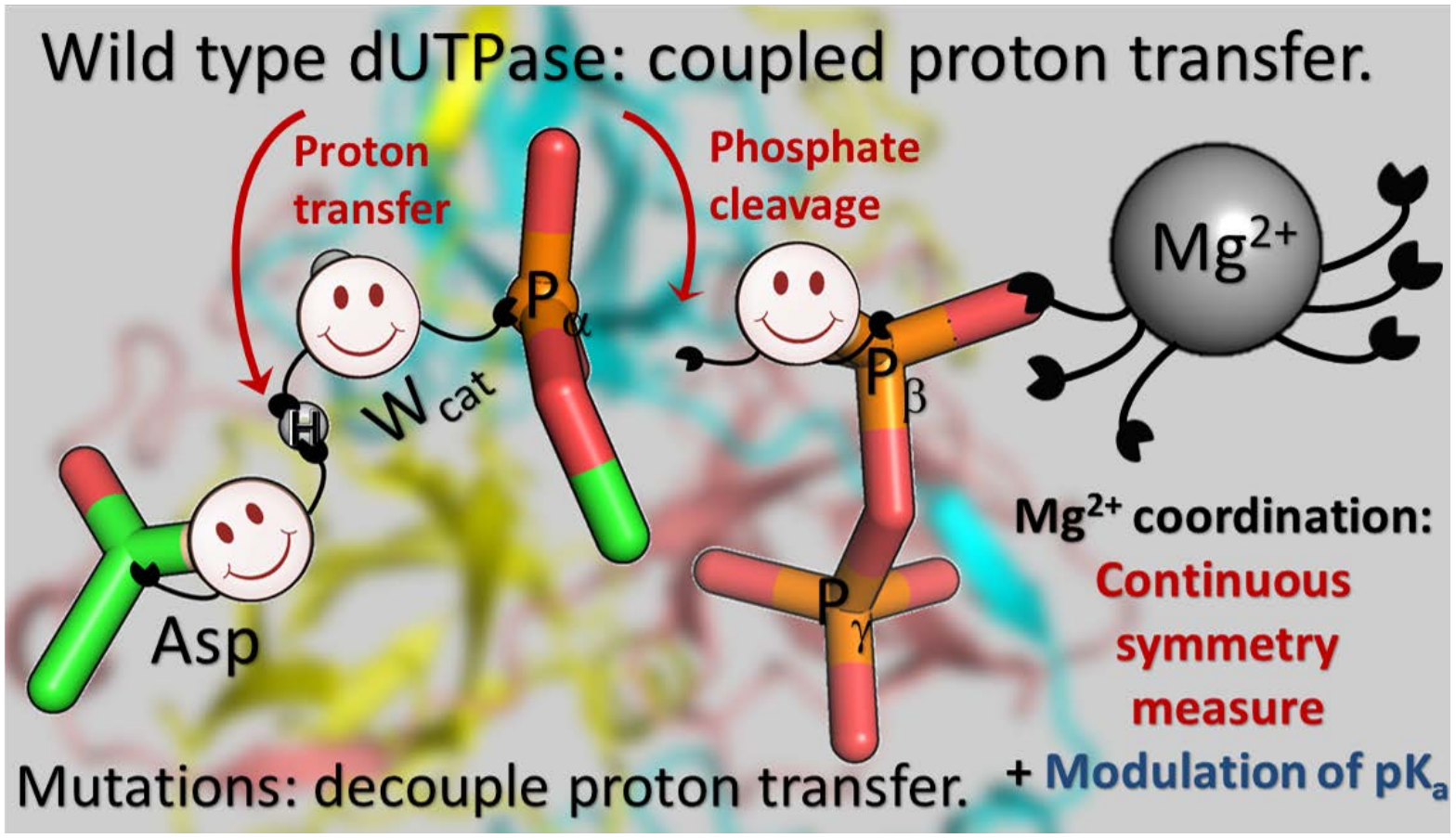

